\title{
Double-diffusive translation of Earth's inner core
}

\author{
R.Deguen, T. Alboussière and S. Labrosse \\ Université de Lyon, UCBL, ENSL, CNRS, LGL-TPE, 69622 Villeurbanne, France. E-mail: renaud.deguen@univ-lyon1.fr
}

Accepted 2018 March 23. Received 2018 March 20; in original form 2017 October 31

\begin{abstract}
SUMMAR Y
The hemispherical asymmetry of the inner core has been interpreted as resulting from a high-viscosity mode of inner core convection, consisting in a translation of the inner core. A thermally driven translation, as originally proposed, is unlikely if the currently favoured high values of the thermal conductivity of iron at core conditions are correct. We consider here the possibility that inner core translation results from an unstable compositional gradient, which would develop either because the light elements present in the core become increasingly incompatible as the inner core grows, or because of a possibly positive feedback of the development of the F-layer on inner core convection. Though the magnitude of the destabilizing effect of the compositional field is predicted to be similar to or smaller than the stabilizing effect of the thermal field, the huge difference between thermal and chemical diffusivities implies that double-diffusive instabilities can still arise even if the net buoyancy increases upward. Using linear stability analysis and numerical simulations, we demonstrate that a translation mode can indeed exist if the compositional field is destabilizing, even if the temperature profile is subadiabatic, and irrespectively of the relative magnitudes of the composition and potential temperature gradients. The existence of this double diffusive mode of translation requires that the following conditions are met: (i) the compositional profile within the inner core is destabilizing, and remains so for a duration longer than the destabilization timescale (on the order of $200 \mathrm{Myr}$, but strongly dependent on the magnitude of the initial perturbation); and (ii) the inner core viscosity is sufficiently large, the required value being a strongly increasing function of the inner core size (e.g. $10^{17} \mathrm{~Pa} \mathrm{~s}$ when the inner core was $200 \mathrm{~km}$ in radius, and $\simeq 3 \times 10^{21} \mathrm{~Pa}$ s at the current inner core size). If these conditions are met, the predicted inner core translation rate is found to be similar to the inner core growth rate, which is more consistent with inferences from the geomagnetic field morphology and secular variation than the higher translation rate predicted for a thermally driven translation.
\end{abstract}

Key words: Core; Instability analysis; Numerical modelling.

\section{INTRODUCTION}

Apart from its North-South anisotropy (Poupinet et al. 1983; Morelli et al. 1986; Woodhouse et al. 1986), the most prominent feature of Earth's inner core is the hemispherical dichotomy observed in its seismological properties: the western hemisphere (west from Greenwich) exhibits stronger anisotropy, lower $P$-wave velocity, and less attenuation than the eastern hemisphere (Tanaka \& Hamaguchi 1997; Niu \& Wen 2001; Garcia 2002; Irving et al. 2009; Deuss et al. 2010; Monnereau et al. 2010; Waszek et al. 2011; Tanaka 2012; Lythgoe et al. 2014). This has been proposed to result from either heterogeneous crystallization due to long-term mantle control (Sumita \& Olson 1999; Aubert et al. 2008; Gubbins et al. 2011) or from a thermal convection mode consisting in a translation of the inner core (Alboussière et al. 2010; Monnereau et al. 2010), which is the focus of this paper. Inner core translation could plausibly explain the hemispherical structure of the inner core, through textural change of the iron aggregate during the translation (Bergman et al. 2010; Monnereau et al. 2010; Cormier et al. 2011; Geballe et al. 2013; Lasbleis et al. 2017; Calvet \& Margerin 2018), but could also have profound implications for the dynamics and structure of the outer core. If fast enough, translation can result in significant melting on one of the inner core hemisphere. This may be at the origin (Alboussière et al. 2010) of the so-called F-layer, a layer of anomalously low $P$-wave velocity gradient (Souriau \& Poupinet 1991) which has been interpreted as an iron-rich, stably stratified layer (Gubbins et al. 2008). Inner core translation, by imposing a highly asymmetric buoyancy flux at the base of the outer core, is also a promizing candidate (Aubert 2013; Aubert et al. 2013; Davies et al. 2013) for explaining the existence of the planetary scale eccentric gyre which has been inferred from quasi-geostrophic core flow inversions (Pais \& Jault 2008; Gillet et al. 2009).

The translation of the inner core proposed by Monnereau et al. (2010) and Alboussière et al. (2010) is a high-viscosity mode of 
thermal convection. Its existence, therefore, requires the temperature profile in the inner core to be superadiabatic. Thermal convection in the inner core may arise because the temperature at the inner core boundary (ICB) decreases with time as the inner core grows. This builds a temperature profile decreasing from the centre outwards, a situation which could lead to convection if the temperature profile is steeper than the adiabat. The temperature profile within the inner core depends on how efficiently heat is extracted from within the inner core, and on the cooling rate at the ICB. Fast inner core growth and low thermal diffusivity promote a steep, possibly superadiabatic temperature profile; slow inner core growth and high thermal diffusivity results in a flatter, subadiabatic temperature profile.

When the thermally driven convective translation of the inner core was proposed, existing experiments and models favoured a rather low value of the inner core thermal conductivity $\left[36 \mathrm{~W} \mathrm{~m}^{-1}\right.$ $\mathrm{K}^{-1}$ according to Stacey \& Davis (2008)] making the existence of thermal convection in the inner core likely. However, the value of the thermal conductivity has since been re-evaluated significantly upward, with all published studies arguing for a thermal conductivity higher than $\sim 100 \mathrm{~W} \mathrm{~m}^{-1} \mathrm{~K}^{-1}$ in the outer core and possibly larger than $200 \mathrm{~W} \mathrm{~m}^{-1} \mathrm{~K}^{-1}$ in the inner core (Sha \& Cohen 2011; de Koker et al. 2012; Pozzo et al. 2012; Gomi et al. 2013; Pozzo et al. 2014; Gomi et al. 2016; Ohta et al. 2016; Zhang et al. 2016), with the exception of Konôpková et al. (2016) who estimate the core thermal conductivity at $18-44 \mathrm{~W} \mathrm{~m}^{-1} \mathrm{~K}^{-1}$. The high thermal conductivity makes thermally driven convection - and translation - in the inner core unlikely (Deguen et al. 2013; Labrosse 2014; Pozzo et al. 2014; Lythgoe et al. 2015).

Compositional convection might be a viable alternative to thermal convection (Gubbins et al. 2013; Labrosse 2014; Pozzo et al. 2014; Lythgoe et al. 2015): an unstable compositional gradient may arise in the inner core either because the light elements present in the core become increasingly incompatible as the inner core grows (Deguen \& Cardin 2011; Gubbins et al. 2013), or because of a possibly positive feedback of the development of the F-layer on inner core convection (Deguen et al. 2013). Modelling of the thermal and compositional evolution of the inner core indicates that the two contributions are similar in magnitude, though the stabilizing thermal effect is likely larger than the destabilizing compositional effect (Labrosse 2014). It is well known that in this configuration, doublediffusive convection can arise owing to the large contrast between the thermal and compositional diffusivities (Stommel et al. 1956; Stern 1960; Huppert \& Turner 1981). The possibility of doublediffusive convection of the inner core has been suggested by Pozzo et al. (2014).

The goal of this paper is to investigate the conditions of existence of inner core translation in a situation where the temperature field is stabilizing (subadiabatic temperature profile) and the composition field destabilizing. The governing equations are presented in Section 2 and numerical methods are briefly discussed in Section 3. A linear stability analysis of the governing equations (Section 4) demonstrates that an instability of double-diffusive type can develop and initiate a translation motion if the compositional field is destabilizing and the temperature field stabilizing. Expressions for the steady-state translation rate are then obtained in Section 5. The deformation induced by lateral temperature and compositional variations is investigated in Section 6. We then use our results to discuss in Section 7 the feasibility of double-diffusive translation of Earth's inner core. The parameters and variables used in this study are summarized in Table 1, with their definitions or values when applicable.

\section{GOVERNING EQUATIONS}

In this section, we present the systems of equations used here to model thermochemical convection in the inner core, with melting and freezing at the ICB. We present both a set of equations for a viscous (with newtonian rheology) inner core, and a second set of equations strictly valid in the limit of a rigid inner core (infinite viscosity), which will be used in Sections 4 and 5 to investigate the conditions of existence of a double-diffusive mode of translation and obtain analytical expressions for the steady-state translation rate. The rigid inner core set of equations generalizes the treatment presented in Alboussière et al. (2010) to take into account time dependance, a compositional field in addition to the thermal field, and non-negligible diffusion.

\subsection{Heat and composition transport}

We use a potential temperature formulation where we define the potential temperature as $\Theta=T(r, \theta, \varphi, t)-T_{a d}(r, t)$, where $T_{a d}(r$, $t$ ) is the adiabatic temperature profile anchored at the ICB (Deguen $\&$ Cardin 2011). Under the assumption that the dissipation number is small (it is about $7 \times 10^{-2}$ in the inner core) but allowing for temperature variations of the same order of magnitude as adiabatic variations, this allows to obtain from the entropy balance the following transport equation for $\Theta$ :

$\frac{\mathrm{D} \Theta}{\mathrm{D} t}=\kappa_{T} \nabla^{2} \Theta+S_{T}, \quad \Theta\left(r_{\mathrm{ic}}\right)=0$,

where $\kappa_{T}$ is the thermal diffusivity and

$S_{T}=-\frac{\partial T_{\mathrm{ad}}}{\partial t}+\kappa_{T} \nabla^{2} T_{\mathrm{ad}}$

is a source term comparing the effects of the secular decrease of the temperature at the ICB, which helps maintaining a temperature difference across the inner core, with conduction of heat along the adiabat.

The equation for the transport of composition can be written in a mathematically equivalent way (Deguen et al. 2013; Lasbleis et al. 2015; Lythgoe et al. 2015) by defining a potential composition $\chi=c_{s}-c_{s}^{\text {icb }}$, where $c_{s}(r, \theta, \varphi, t)$ is the concentration of a given element in the inner core and $c_{s}^{\text {icb }}$ is its concentration at the ICB on the solid side. We denote by $c_{l}^{\text {icb }}$ the concentration on the liquid side of the ICB, assumed to be a function of time only, and by $k=$ $c_{s}^{\text {icb }} / c_{l}^{\text {icb }}$ the equilibrium partition coefficient of this light element at the ICB conditions. With this formulation, the equation of transport of composition writes

$\frac{\mathrm{D} \chi}{\mathrm{D} t}=\kappa_{c} \nabla^{2} \chi+S_{c}, \quad \chi\left(r_{\mathrm{ic}}\right)=0$,

where $\kappa_{c}$ is the compositional diffusivity and

$S_{c}=-\frac{\mathrm{d} c_{s}^{\mathrm{icb}}}{\mathrm{d} t}=-k \frac{\mathrm{d} c_{l}^{\mathrm{icb}}}{\mathrm{d} t}-c_{l}^{\mathrm{icb}} \frac{\mathrm{d} k}{\mathrm{~d} t}$.

In the few calculations taking into account the growth of the inner core (Section 7.4), we use a front fixing method which in effect introduces in eqs (1) and (3) an additional radial advection term equal to $\dot{r}_{\text {ic }}\left(r / r_{\text {ic }}\right) \partial \Theta / \partial r$ and $\dot{r}_{\text {ic }}\left(r / r_{\text {ic }}\right) \partial \chi / \partial r$, respectively, where $r_{\text {ic }}(t)$ is the radius of the inner core at time $t$ and $\dot{r}_{\text {ic }}=d r_{\text {ic }} / d t$ is the inner core growth rate. For simplicity, we will only present in what follows a set of equations assuming a constant inner core radius. All the details related to properly taking into account inner core growth can be found in Deguen \& Cardin (2011). 
Table 1. Definitions of the main parameters and variables used in this study, with representative values or definition when applicable.

\begin{tabular}{|c|c|c|c|c|c|}
\hline Present-day inner core radius ${ }^{a}$ & $r_{\mathrm{ic}}^{p}$ & $1221 \mathrm{~km}$ & & & \\
\hline Density in the inner core ${ }^{a}$ & $\rho$ & $13000 \mathrm{~kg} \mathrm{~m}^{-3}$ & & & \\
\hline Density jump at the $\mathrm{ICB}^{a}$ & $\Delta \rho$ & $600 \mathrm{~kg} \mathrm{~m}^{-3}$ & & & \\
\hline Gravitational acceleration $^{a}$ & $g$ & $4.4 \mathrm{~m}^{2} \mathrm{~s}^{-1}$ at the ICB & & & \\
\hline Radial gravity gradient ${ }^{a}$ & $g^{\prime}=\mathrm{d} g / \mathrm{d} r$ & $3.6 \times 10^{-6} \mathrm{~s}^{-2}$ & & & \\
\hline Isentropic bulk modulus $^{a}$ & $K_{S}$ & $1400 \mathrm{GPa}$ & & & \\
\hline Dynamic viscosity ${ }^{b}$ & $\eta$ & $10^{11}$ to $10^{22} \mathrm{~Pa} \mathrm{~s}$ & & & \\
\hline \multicolumn{2}{|c|}{ Thermal parameters and variables } & & \multicolumn{2}{|c|}{ Compositional parameters and variables } & \\
\hline Temperature & $T$ & & Concentration (solid, liquid) & $c_{S}, c_{l}$ & \\
\hline Adiabat & $T_{\mathrm{ad}}$ & & $\begin{array}{l}\text { Solid/liquid partition } \\
\text { coefficient }\end{array}$ & $k=c_{s}^{\mathrm{icb}} / c_{l}^{\mathrm{icb}}$ & \\
\hline Potential temperature & $\Theta=T-T_{\mathrm{ad}}$ & & Potential composition & $\chi=c_{s}-c_{s}^{\mathrm{icb}}$ & \\
\hline Thermal diffusivity $^{c}$ & $\kappa_{T}$ & $0.5-2 \times 10^{-5} \mathrm{~m}^{2} \mathrm{~s}^{-1}$ & Compositional diffusivity ${ }^{f}$ & $\kappa_{c}$ & $10^{-12} \mathrm{~m}^{2} \mathrm{~s}^{-1}$ \\
\hline $\begin{array}{l}\text { Thermal expansion } \\
\text { coefficient }^{d}\end{array}$ & $\alpha_{T}$ & $10^{-5} \mathrm{~K}^{-1}$ & $\begin{array}{l}\text { Compositional expansion } \\
\text { coefficient } f^{f}\end{array}$ & $\alpha_{c}$ & 1 \\
\hline Thermal source term & $S_{T}$ & eq. (2) & Compositional source term & $S_{c}$ & eq. (4) \\
\hline Gruneisen parameter $^{d}$ & $\gamma$ & 1.4 & & & \\
\hline Clapeyron/adiabat slopes ratio ${ }^{e}$ & $\mathrm{~d} T_{s} / \mathrm{d} T_{\mathrm{ad}}$ & 1.65 & & & \\
\hline \multicolumn{6}{|c|}{ Relevant timescales } \\
\hline Phase change timescale & $\tau_{\varphi}$ & $\sim 1 \mathrm{ky}$ & & & \\
\hline Viscous relaxation timescale & $\tau_{\eta}$ & $\eta /\left(\Delta \rho g^{\prime} r_{\mathrm{ic}}^{2}\right) \sim 5 \min$ & to $0.1 \mathrm{My}$ & & \\
\hline Thermal diffusion timescale ${ }^{g}$ & $\tau_{\kappa_{T}}$ & $r_{\mathrm{ic}}^{2} / \kappa_{T} \sim 5 \mathrm{~Gy}$ & & & \\
\hline $\begin{array}{l}\text { Compositional diffusion } \\
\text { timescale }^{g}\end{array}$ & $\tau_{\kappa_{c}}$ & $r_{\mathrm{ic}}^{2} / \kappa_{c} \sim 5 \times 10^{7} \mathrm{~Gy}$ & & & \\
\hline Advection timescale & $\tau_{V}$ & $r_{\mathrm{ic}} / V$ & & & \\
\hline \multicolumn{6}{|c|}{ Non-dimensional parameters } \\
\hline Phase change number & $\mathcal{P}$ & $\tau_{\varphi} / \tau_{\eta}$ & & & \\
\hline Lewis number & Le & $\kappa_{T} / \kappa_{c}=\tau_{\kappa_{c}} / \tau_{\kappa_{T}} \sim 10$ & & & \\
\hline Buoyancy ratio & $\mathcal{B}$ & $\alpha_{T} S_{T} /\left(\alpha_{c} S_{c}\right)$ & & & \\
\hline Thermal Rayleigh number & $R a_{\Theta}$ & eq. (9) & $\begin{array}{l}\text { Compositional Rayleigh } \\
\text { number }\end{array}$ & $R a_{\chi}$ & eq. (9) \\
\hline Thermal Péclet number & $\begin{array}{l}\mathcal{R}_{\Theta}=R a_{\Theta} / \mathcal{P} \\
P e_{T}\end{array}$ & $\tau_{\kappa_{T}} / \tau_{V}$ & Compositional Péclet number & $\begin{array}{l}\mathcal{R}_{\chi}=R a_{\chi} / \mathcal{P} \\
P e_{c}\end{array}$ & $\tau_{\kappa_{c}} / \tau_{V}$ \\
\hline
\end{tabular}

${ }^{a}$ From PREM (Dziewonski \& Anderson 1981).

${ }^{b}$ Yoshida et al. (1996); Buffett (1997); Van Orman (2004); Mound \& Buffett (2006); Koot \& Dumberry (2011); Reaman et al. (2011, 2012).

${ }^{c}$ corresponding to a thermal conductivity between 50 and $200 \mathrm{~W} \mathrm{~m}^{-1} \mathrm{~K}^{-1}$ (Sha \& Cohen 2011; de Koker et al. 2012; Pozzo et al. 2012; Gomi et al. 2013; Pozzo et al. 2014; Gomi et al. 2016; Konôpková et al. 2016; Ohta et al. 2016; Zhang et al. 2016).

${ }^{d}$ Vocadlo (2015).

${ }^{e}$ Deguen \& Cardin (2011).

${ }^{f}$ Gubbins et al. (2013).

${ }^{g}$ The free-decay timescale of a spherically symmetric field and of a degree 1 heterogeneity in a sphere are respectively $r_{\text {ic }}^{2} /\left(\alpha_{01}^{2} \kappa\right)$ and $r_{\text {ic }}^{2} /\left(\alpha_{11}^{2} \kappa\right)$, where $\alpha_{01}=\pi$ and $\alpha_{11} \simeq 4.4934$ are the first zeros of the degree 0 and degree 1 spherical Bessel function of the first kind. This is respectively a factor 9.87 and 20.2 smaller than the timescales given in the table.

\subsection{Dimensionless equations}

We denote by $\Delta \Theta$ the difference of potential temperature across the inner core, and by $\Delta \chi$ the difference of potential composition across the inner core. The acceleration of gravity is taken to vary linearly with radius, $g(r)=g^{\prime} r$. Scaling lengths by $r_{\text {ic }}$, time by $r_{\text {ic }}^{2} / \kappa_{T}$, velocity by $\kappa_{T} / r_{\text {ic }}$, temperature by $\Delta \Theta$, and composition by $\Delta \chi$, the equations of conservation of momentum, continuity, heat and composition transport write

$0=-\nabla p+\left(R a_{\Theta} \Theta+\frac{R a_{\chi}}{L e} \chi\right) \mathbf{r}+\nabla^{2} \mathbf{u}$

$\nabla \cdot \mathbf{u}=0$

$\frac{\mathrm{D} \Theta}{\mathrm{D} t}=\nabla^{2} \Theta+\frac{S_{T} r_{\mathrm{ic}}^{2}}{\kappa_{T} \Delta \Theta}$,
$L e \frac{\mathrm{D} \chi}{\mathrm{D} t}=\nabla^{2} \chi+\frac{S_{c} r_{\mathrm{ic}}^{2}}{\kappa_{c} \Delta \chi}$

where $p$ is the dynamic pressure, $\mathbf{u}$ the velocity, $\mathbf{r}=r \mathbf{e}_{r}$. The thermal and compositional Rayleigh numbers and the Lewis number are defined as

$R a_{\Theta}=\frac{\alpha_{T} \rho g^{\prime} \Delta \Theta r_{\mathrm{ic}}^{4}}{\kappa_{T} \eta}, R a_{\chi}=\frac{\alpha_{c} \rho g^{\prime} \Delta \chi r_{\mathrm{ic}}^{4}}{\kappa_{c} \eta}, L e=\frac{\kappa_{T}}{\kappa_{c}}$,

where $\alpha_{T}$ and $\alpha_{c}$ are the thermal and compositional expansion coefficients, $\rho$ the density of the inner core and $\eta$ the dynamic viscosity.

Following Alboussière et al. (2010), we assume that melting and freezing happens at the ICB at a rate proportional to the topography $h$ of the ICB, and inversely proportional to a timescale $\tau_{\varphi}$ which depends on how efficiently convection in the outer core is able to transport the latent heat associated with melting or freezing. If the topography varies slowly with time compared to $\tau_{\varphi}$ [estimated to be $\sim 1$ kyr (Alboussière et al. 2010)], this amounts to state that the 
radial velocity $u_{r}$ at the ICB is

$u_{r}=\frac{h}{\tau_{\phi}}$.

Using this phase change law to relate the radial velocity at the ICB to the topography, the continuity of normal stress across the ICB can be written as

$-2 \eta \frac{\partial u_{r}}{\partial r}+p=\Delta \rho g\left(r_{\text {ic }}\right) \tau_{\phi} u_{r}$

(Deguen et al. 2013), where $\Delta \rho$ is the density jump across the ICB. Once made dimensionless, this can be written as

$-2 \frac{\partial u_{r}}{\partial r}+p=\mathcal{P} u_{r}$

where

$\mathcal{P}=\frac{\Delta \rho g^{\prime} r_{i c}^{2} \tau_{\phi}}{\eta}$

is the 'phase change number' introduced in Deguen et al. (2013), which quantifies the resistance to phase change at the ICB. $\mathcal{P}$ is the ratio of $\tau_{\varphi}$ to the timescale $\eta /\left(\Delta \rho g\left(r_{i c}\right) r_{i c}\right)$, which is best understood in this context as the time needed to build from zero a topography balancing the internal stresses: Consider a flow within the inner core with a radial velocity $u_{r}$ varying radially over the lengthscale $r_{\text {ic }}$. Starting from a state with no topography, this flow will deform the ICB up to a point where the normal stress at $r=r_{i c}$, which is $\sim \eta u_{r} / r_{\text {ic }}$, is balanced by the topography weight $\sim \Delta \rho g\left(r_{i c}\right) h$. This happens when $h \sim \eta u_{r} /\left(\Delta \rho g\left(r_{i c}\right) r_{\text {ic }}\right)$. The time needed to build this topography is $h / u_{r} \sim \eta /\left(\Delta \rho g\left(r_{i c}\right) r_{\text {ic }}\right)$.

In the limit of infinite $\mathcal{P}$, the boundary condition (12) reduces to the condition $u_{r}=0$, which corresponds to impermeable conditions. In this limit, the timescale of topography building is short compared to the phase change timescale, which implies the formation of a topography balancing the internal stresses.

In contrast, when $\mathcal{P} \rightarrow 0$, eq. (12) implies that the normal stress tends towards 0 at the ICB, which corresponds to fully permeable boundary conditions. In this limit, the timescale of topography building is long compared to the phase change timescale, which implies that phase change is too fast to allow the development of a topography balancing the internal stresses.

The general case of finite $\mathcal{P}$ gives boundary conditions for which the rate of phase change at the boundary (equal to $u_{r}$ ) is proportional to the normal stress induced by convection within the inner core.

We further impose shear stress free conditions. By construction, $\Theta=\chi=0$ at the ICB.

\subsection{The rigid inner core limit}

In the limit of a rigid inner core (no deformation allowed), the possible motions of the inner core are restricted to either rotational motions (which we will not consider here) and translation motions, which can arise in response to degree 1 density heterogeneities in the inner core.

An expression for the translation rate can be obtained by combining the phase change law eq. (10) with the force balance on the inner core, which we use to obtain the displacement of the inner core from a reference state as a function of the internal density distribution. The force balance on the inner core comes down to an equilibrium between buoyancy forces associated with density anomalies within the inner core and pressure forces applied by the outer core on the inner core surface. This force balance gives the displacement of the inner core and the degree 1 component $h_{1}$ of the ICB topography.
Using the phase change law eq. (10) to relate the translation rate $V$ to $h_{1}$ through $V=h_{1} / \tau_{\varphi}$, we obtain the following expression for the translation rate:

$V=\frac{\rho}{\Delta \rho r_{\mathrm{ic}}^{3} \tau_{\phi}} \int_{0}^{r_{i c b}}\left[\alpha_{T} \Theta_{1}(r)+\alpha_{c} \chi_{1}(r)\right] r^{3} \mathrm{~d} r$

(see Appendix A1 for the derivation). Once made dimensionless, this writes

$V=\int_{0}^{1}\left(\mathcal{R}_{\Theta} \Theta_{1}+\frac{\mathcal{R}_{\chi}}{L e} \chi_{1}\right) r^{3} \mathrm{~d} r$

where

$\mathcal{R}_{\Theta}=\frac{\alpha_{T} \rho \Delta \Theta}{\Delta \rho} \frac{r_{\mathrm{ic}}^{2} / \kappa_{T}}{\tau_{\phi}}=\frac{R a_{\Theta}}{\mathcal{P}}$,

$\mathcal{R}_{\chi}=\frac{\alpha_{c} \rho \Delta \chi}{\Delta \rho} \frac{r_{\text {ic }}^{2} / \kappa_{c}}{\tau_{\phi}}=\frac{R a_{\chi}}{\mathcal{P}}$.

$\mathcal{R}_{\Theta}$ and $\mathcal{R}_{\chi}$ are independent of the viscosity, as appropriately defined non-dimensional numbers should be since here no deformation is allowed. Note that they are also independent of the acceleration of gravity.

In the translation mode, the potential temperature and composition fields are symmetric about the translation direction, and the equations of transport of heat and composition are given by eqs (1) and (3) in which advective transport is restricted to the translation direction:

$\frac{\partial \Theta}{\partial t}+\mathbf{V} \cdot \nabla \Theta=\nabla^{2} \Theta+\frac{S_{T} r_{\mathrm{ic}}^{2}}{\kappa_{T} \Delta \Theta}, \quad \Theta\left(r_{\mathrm{ic}}\right)=0$,

$L e\left(\frac{\partial \chi}{\partial t}+\mathbf{V} \cdot \nabla \chi\right)=\nabla^{2} \chi+\frac{S_{c} r_{\text {ic }}^{2}}{\kappa_{c} \Delta \chi}, \quad \chi\left(r_{\text {ic }}\right)=0$,

where $\mathbf{V}$ is the translation velocity. Expanding $\Theta$ and $\chi$ as series of Legendre polynomials of $\cos \theta$, where $\theta$ is the angle between $\mathbf{r}$ and the translation direction, eqs (17) and (18) can then be written, as demonstrated in Appendix A2, as

$$
\begin{aligned}
\frac{\partial \Theta_{l}}{\partial t}= & -V \frac{l+1}{2 l+3}\left(\frac{l+2}{r}+\frac{\partial}{\partial r}\right) \Theta_{l+1} \\
& +V \frac{l}{2 l-1}\left(\frac{l-1}{r}-\frac{\partial}{\partial r}\right) \Theta_{l-1} \\
& +\mathcal{D}_{l} \Theta_{l}+\frac{S_{T} r_{\mathrm{ic}}^{2}}{\kappa_{T} \Delta \Theta} \delta_{0 l}, \quad \forall l \geq 0, \\
\frac{\partial \chi_{l}}{\partial t}= & -V \frac{l+1}{2 l+3}\left(\frac{l+2}{r}+\frac{\partial}{\partial r}\right) \chi_{l+1} \\
& +V \frac{l}{2 l-1}\left(\frac{l-1}{r}-\frac{\partial}{\partial r}\right) \chi_{l-1} \\
& +\frac{1}{L e}\left(\mathcal{D}_{l} \chi_{l}+\frac{S_{c} r_{\mathrm{ic}}^{2}}{\kappa_{c} \Delta \chi} \delta_{0 l}\right), \quad \forall l \geq 0,
\end{aligned}
$$

which have to be solved together with the boundary conditions $\Theta_{l}(r=1)=\chi_{l}(r=1)=0$. The operator $\mathcal{D}_{l}$ is defined as

$\mathcal{D}_{l}=\frac{\mathrm{d}^{2}}{\mathrm{~d} r^{2}}+\frac{2}{r} \frac{\mathrm{d}}{\mathrm{d} r}-\frac{l(l+1)}{r^{2}}$.

\section{NUMERICAL METHODS}

In the following sections, we will perform a linear stability analysis of double-diffusive convection with a phase-change interface 
(Section 4), derive analytical solutions for the steady-state translation rate (Section 5) and quantify the conditions of existence of the translation mode (Section 6). The theoretical predictions will be compared with numerical simulations obtained by running two different codes: the first one [an extension of the code used in Deguen et al. (2013)] solves the Stokes equation and the temperature and composition transport equations, and has been used here in an axisymmetric configuration; the second is a simpler code only allowing the translation mode, the evolution of which is obtained by solving eqs (15), (19) and (20). The latter approach has the advantage of being highly efficient (the Stokes equation does not need to be solved, and the nonlinear term of the advection equations is solved in the spectral domain).

Up to 512 radial levels and maximal harmonic degree of 256 have been used. Care has been taken to always correctly resolve the compositional boundary layer below the ICB, which can be quite thin.

\section{ONSET OF DOUBLE-DIFFUSIVE CONVECTION}

\subsection{Marginal stability}

We consider here an initial state at rest, with the potential temperature and composition fields following spherically symmetric profiles $\bar{\Theta}(r)$ and $\bar{\chi}(r)$. This state is perturbed by infinitesimal perturbations $\Theta^{\prime}(r, \theta, \varphi, t)$ and $\chi^{\prime}(r, \theta, \varphi, t)$, and we write

$\Theta(r, \theta, \phi, t)=\bar{\Theta}(r)+\Theta^{\prime}(r, \theta, \phi, t)$,

$\chi(r, \theta, \phi, t)=\bar{\chi}(r)+\chi^{\prime}(r, \theta, \phi, t)$.

The evolution of the perturbations is controlled by the equations of conservation of momentum, heat and composition given by eqs (5), (1) and (3).

Taking twice the curl of the Stokes, eq. (5) gives

$0=-\nabla^{2}\left(R a_{\Theta} \Theta^{\prime}+\frac{R a_{\chi}}{L e} \chi^{\prime}\right) \mathbf{r}-\nabla^{4} \mathbf{u}$,

where $\nabla^{4}(\ldots)=\nabla \times \nabla \times \nabla \times \nabla \times(\ldots)$. Linearizing the heat and composition transport equations gives

$u_{r} \frac{\partial \bar{\Theta}}{\partial r}=\nabla^{2} \Theta^{\prime}$,

$u_{r} \frac{\partial \bar{\chi}}{\partial r}=\frac{1}{L e} \nabla^{2} \chi^{\prime}$

Multiplying eq. (25) by $R a_{\Theta}$, eq. (26) by $R a_{\chi}$ and adding the two equations, gives

$u_{r}\left(R a_{\Theta} \frac{\partial \bar{\Theta}}{\partial r}+R a_{\chi} \frac{\partial \bar{\chi}}{\partial r}\right)=\nabla^{2}\left(R a_{\Theta} \Theta^{\prime}+\frac{R a_{\chi}}{L e} \chi^{\prime}\right)$,

which we inject into eq. (24) to obtain

$0=u_{r}\left(R a_{\Theta} \frac{\partial \bar{\Theta}}{\partial r}+R a_{\chi} \frac{\partial \bar{\chi}}{\partial r}\right) \mathbf{r}+\nabla^{4} \mathbf{u}$.

This can be solved with the boundary conditions given by the continuity of stress at the ICB (eq. 12 plus shear-stress-free conditions).

Assuming for illustration that the base profiles $\bar{\Theta}$ and $\bar{\chi}$ have the same $r$ dependency, this gives the exact same equation as would have been obtained in the case of purely thermal convection, with
$R a_{\Theta}+R a_{\chi}$ instead of $R a_{\Theta}$. In this case, the results on the marginal stability of thermal convection in the inner core obtained in Deguen et al. (2013) can be extended to the case of two-components convection by simply replacing $R a_{\Theta}$ by $R a_{\Theta}+R a_{\chi}$. Instability requires that

$R a_{\Theta}+R a_{\chi}>R a_{\mathrm{cr}}(\mathcal{P})$,

where the critical Rayleigh number $R a_{\mathrm{cr}}$ is a function of $\mathcal{P}$, which can be calculated numerically as explained in Deguen et al. (2013). $R a_{\text {cr }}$ is well approximated by

$R a_{\text {cr }}= \begin{cases}\frac{175}{2} \mathcal{P} & \text { if } \mathcal{P} \ll 10^{2}, \\ 1545.6 & \text { if } \mathcal{P} \gg 10^{2} .\end{cases}$

Eq. (29) demonstrates that the convection instability can develop even when the sum of the thermal and compositional buoyancy $\alpha_{T} \Theta+\alpha_{c} \chi$ increases upward: since $\kappa_{c} \ll \kappa_{T}, R a_{\Theta}+R a_{\chi}$ can be positive with $R a_{\Theta}<0(\Delta \Theta<0)$ even if $\left|\alpha_{T} \Delta \Theta\right|>\left|\alpha_{c} \Delta \chi\right|$. This is typical of double-diffusive convection, and a direct consequence of the differential diffusion of the thermal and compositional fields: convective motions can develop because the much larger thermal diffusivity allows temperature heterogeneities to diffuse much faster than compositional heterogeneities.

The mathematical analogy at the marginal state between the twocomponents case and the thermal case also holds when calculating the first unstable mode. The analysis of Deguen et al. (2013; Section 4), thus, implies that the first unstable mode consists in a translation if $\mathcal{P} \lesssim 30$. In this limit, taking $R a_{c r}=\frac{175}{2} \mathcal{P}$ and dividing eq. (29) by $\mathcal{P}$ gives the following criterion for instability:

$\mathcal{R}_{\Theta}+\mathcal{R}_{\chi}>\frac{175}{2}$.

Again, the instability criterion can be met even when $\alpha_{T} \Theta+\alpha_{c} \chi$ increases upwards if $\kappa_{c} \ll \kappa_{T}$, suggesting that a double-diffusive translation mode is possible.

\subsection{The $\mathcal{P} \rightarrow 0$ limit}

\subsubsection{Marginal stability}

If we restrict ourselves to the emergence of the translation mode, the critical value of $\mathcal{R}_{\Theta}$ and $\mathcal{R}_{\chi}$ for more general forms of the potential temperature and composition profiles can be found by analysing the set of equations developed in Section 2.3 for a pure translation. The inner core is unstable against the translation instability if any arbitrarily small perturbations of the degree one components of the thermal and compositional fields initially grow with time, that is, if

$\frac{1}{\Theta_{1}} \frac{\partial \Theta_{1}}{\partial t}=\frac{1}{\Theta_{1}}\left[-V \frac{2}{5}\left(\frac{3}{r}+\frac{\partial}{\partial r}\right) \Theta_{2}-V \frac{\partial \Theta_{0}}{\partial r}+\mathcal{D}_{1} \Theta_{1}\right]>0$,

$\frac{1}{\chi_{1}} \frac{\partial \chi_{1}}{\partial t}=\frac{1}{\chi_{1}}\left[-V \frac{2}{5}\left(\frac{3}{r}+\frac{\partial}{\partial r}\right) \chi_{2}-V \frac{\partial \chi_{0}}{\partial r}+\frac{\mathcal{D}_{1} \chi_{1}}{L e}\right]>0$.

The marginal stability state (neither growth or decay of any infinitesimal perturbations) corresponds to $\partial \Theta_{1} / \partial t=\partial \chi_{1} / \partial t=0$. Linearizing eqs (32) and (33) and keeping only terms of order 1 in perturbation quantities (i.e. ignoring the terms in $V \Theta_{2}$ and $V \chi_{2}$ ), the marginal stability state corresponds to

$0=-V \frac{\partial \Theta_{0}}{\partial r}+\mathcal{D}_{1} \Theta_{1}$, 
$0=-V \frac{\partial \chi_{0}}{\partial r}+\frac{1}{L e} \mathcal{D}_{1} \chi_{1}$.

At this stage, we let the mathematical forms of $\Theta_{0}$ and $\chi_{0}$ unspecified and just write

$\Theta_{1}=V \mathcal{D}_{1}^{-1}\left\{\frac{\partial \Theta_{0}}{\partial r}\right\} \quad$ and $\quad \chi_{1}=\operatorname{LeV} \mathcal{D}_{1}^{-1}\left\{\frac{\partial \chi_{0}}{\partial r}\right\}$

the solutions of eqs (34) and (35) which vanish at $r=1$, as must $\Theta_{1}$ and $\chi_{1}$. Injecting these expressions in eq. (15) gives the following relation between $\mathcal{R}_{\Theta}$ and $\mathcal{R}_{\chi}$ at marginal stability:

$\mathcal{R}_{\Theta} \int_{0}^{1} \mathcal{D}_{1}^{-1}\left\{\frac{\partial \Theta_{0}}{\partial r}\right\} r^{3} \mathrm{~d} r+\mathcal{R}_{\chi} \int_{0}^{1} \mathcal{D}_{1}^{-1}\left\{\frac{\partial \chi_{0}}{\partial r}\right\} r^{3} \mathrm{~d} r=1$.

The critical values of $\mathcal{R}_{\Theta}$ and $\mathcal{R}_{\chi}$ corresponding to either purely thermal or purely compositional convection are given by

$\mathcal{R}_{\Theta, \text { cr }}=\left[\int_{0}^{1} \mathcal{D}_{1}^{-1}\left\{\frac{\partial \Theta_{0}}{\partial r}\right\} r^{3} \mathrm{~d} r\right]^{-1}$,

$\mathcal{R}_{\chi, \mathrm{cr}}=\left[\int_{0}^{1} \mathcal{D}_{1}^{-1}\left\{\frac{\partial \chi_{0}}{\partial r}\right\} r^{3} \mathrm{~d} r\right]^{-1}$,

which will be calculated explicitly below for given forms of the temperature and compositional profiles. Convection sets in if

$\frac{\mathcal{R}_{\Theta}}{\mathcal{R}_{\Theta, \mathrm{cr}}}+\frac{\mathcal{R}_{\chi}}{\mathcal{R}_{\chi, \mathrm{cr}}}>1$.

Let us now evaluate $\mathcal{R}_{\Theta, \text { cr }}$ and $\mathcal{R}_{\chi, \text { cr }}$ for different forms of the thermal or compositional profiles. Taking into account the variation of the partition coefficient with temperature (Gubbins et al. 2013), Labrosse (2014) found that the composition profile in the inner core is well approximated by

$\chi_{0}=\frac{\frac{3}{2} r_{\mathrm{ic}}^{*}\left(1-r^{2}\right)-\left(1-r^{3}\right)}{\frac{3}{2} r_{\mathrm{ic}}^{*}-1}$,

where $r_{\mathrm{ic}}^{*}$ is the inner core radius (here normalized by the current inner core radius) at which the effect of the secular enrichment of the outer core in incompatible elements becomes dominant over the effect of the variation of the partition coefficient with temperature. The radial composition gradient is then given by

$\frac{\partial \chi_{0}}{\partial r}=-2 \frac{r^{2}-r_{\mathrm{ic}}^{*} r}{2 / 3-r_{\mathrm{ic}}^{*}}$.

The critical value of $\mathcal{R}_{\chi}$ is thus given by

$\mathcal{R}_{\chi, \mathrm{cr}}=\frac{r_{\mathrm{ic}}^{*}-2 / 3}{2}\left[\int_{0}^{1} \mathcal{D}_{1}^{-1}\left\{r^{2}-r_{\mathrm{ic}}^{*} r\right\} r^{3} \mathrm{~d} r\right]^{-1}$.

It can be verified that, for any number $\alpha$, the solution of the equation $\mathcal{D}_{1}(x)=r^{\alpha}$ which vanishes at $r=1$ is

$\mathcal{D}_{1}^{-1}\left(r^{\alpha}\right)=\frac{r^{\alpha+2}-r}{\alpha^{2}+5 \alpha+4}$.

Using this expression, we find from eq. (43) that

$\mathcal{R}_{\chi, \mathrm{cr}}=80 \frac{\frac{3}{2} r_{\mathrm{ic}}^{*}-1}{\frac{48}{35} r_{\mathrm{ic}}^{*}-1}$.

The case of a constant partition coefficient $k$ corresponds to the limit $r_{\mathrm{ic}}^{*}=0$ in eq. (45), which gives

$\mathcal{R}_{\chi, \mathrm{cr}}=80$.
In the thermal case, the basic potential temperature profile is set by an equilibrium between cooling and thermal diffusion (Deguen $\&$ Cardin 2011). The potential temperature difference across the inner core is given by $\Delta \Theta=S_{T} r_{\text {ic }}^{2} /\left(6 \kappa_{T}\right)$, and the non-dimensional temperature profile is given by $1-r^{2}$, which corresponds mathematically to the limit $r_{\mathrm{ic}}^{*} \rightarrow \infty$ in eq. (45). This gives

$\mathcal{R}_{\Theta, \mathrm{cr}}=\frac{175}{2}=87.5$

in agreement with the value found by Deguen et al. (2013) from a more general linear stability analysis (not restricted to the translation mode).

In the case of a positive $\mathcal{R}_{\chi}$ and negative $\mathcal{R}_{\Theta}$, the instability condition eq. (40) can be recast as

$\frac{\alpha_{c} \Delta \chi}{-\alpha_{T} \Delta \Theta}>\left(\frac{\mathcal{R}_{\chi, \mathrm{cr}}}{\mathcal{R}_{\Theta, \mathrm{cr}}}-\frac{\mathcal{R}_{\chi, \mathrm{cr}}}{\mathcal{R}_{\Theta}}\right) L e^{-1}$.

$\mathcal{R}_{\Theta}$ and $\mathcal{R}_{\chi}$ are both expected to be large in magnitude compared with their critical values. Since in addition the critical values $\mathcal{R}_{\Theta, \text { cr }}$ and $\mathcal{R}_{\chi, \text { cr }}$ are of the same order of magnitude, the condition for instability in practice reduces to

$\frac{-\alpha_{T} \Delta \Theta}{\alpha_{c} \Delta \chi} \lesssim L e$.

$L e \simeq 2 \times 10^{7}$ in the inner core (Gubbins et al. 2013), which implies that the inner core can be unstable against the translation instability even if the magnitude of the destabilizing compositional density gradient is much smaller than a stabilizing thermal density gradient. Labrosse (2014) obtained values of $\frac{\alpha_{T} \Delta \Theta}{\alpha_{c} \Delta \chi}$ ranging from about -5 to positive values, depending on the outer core composition and assumptions on the evolution of the core. In what follows, we will take -3 as a representative value of the stabilizing thermal stratification/destabilizing compositional stratification case.

\subsubsection{Growth rate of the translation instability}

Though instability appears almost unavoidable when the compositional profile is destabilizing, whether the translation rate eventually becomes significant depends on the growth rate $\sigma$ of the instability compared to the inner core age, and on the magnitude of the initial perturbations. It is, therefore, useful to estimate the growth rate of the instability when the conditions are supercritical. This is done in Appendix A3, where the growth rate is obtained by decomposing the spherical harmonics degree 1 component of the composition and temperature fields as series of spherical Bessel functions, before solving numerically the linear system of equations obtained from the linearized conservation equations and boundary conditions.

Fig. 1 shows with thick solid lines the growth rate of the translation instability as a function of $\mathcal{R}_{\chi}$, with $\frac{\alpha_{T} \Delta \Theta}{\alpha_{c} \Delta \chi}$ fixed at $-3, L e=10^{4}$ or $2 \times 10^{7}$, and different forms of the base compositional profile ( $r_{\text {ic }}^{*}=0,1$, and $\infty$ in eq. (41)). When $\mathcal{R}_{\chi}$ is increased above the critical value for instability, $\sigma$ initially increases in proportion to $\mathcal{R}_{\chi}$ before reaching a plateau.

In the limit of $L e \gg 1$, a good approximation of the growth rate $\sigma$ of the translation instability is given (in dimensional form) by

$\sigma=\frac{\kappa_{T}}{r_{\mathrm{ic}}^{2}} \frac{1 / 3-2 / 5 r_{\mathrm{ic}}^{*}}{2 / 3-r_{\mathrm{ic}}^{*}} \frac{\mathcal{R}_{\chi} / L e}{1-\frac{\mathcal{R}_{\Theta}}{175 / 2}}$ 

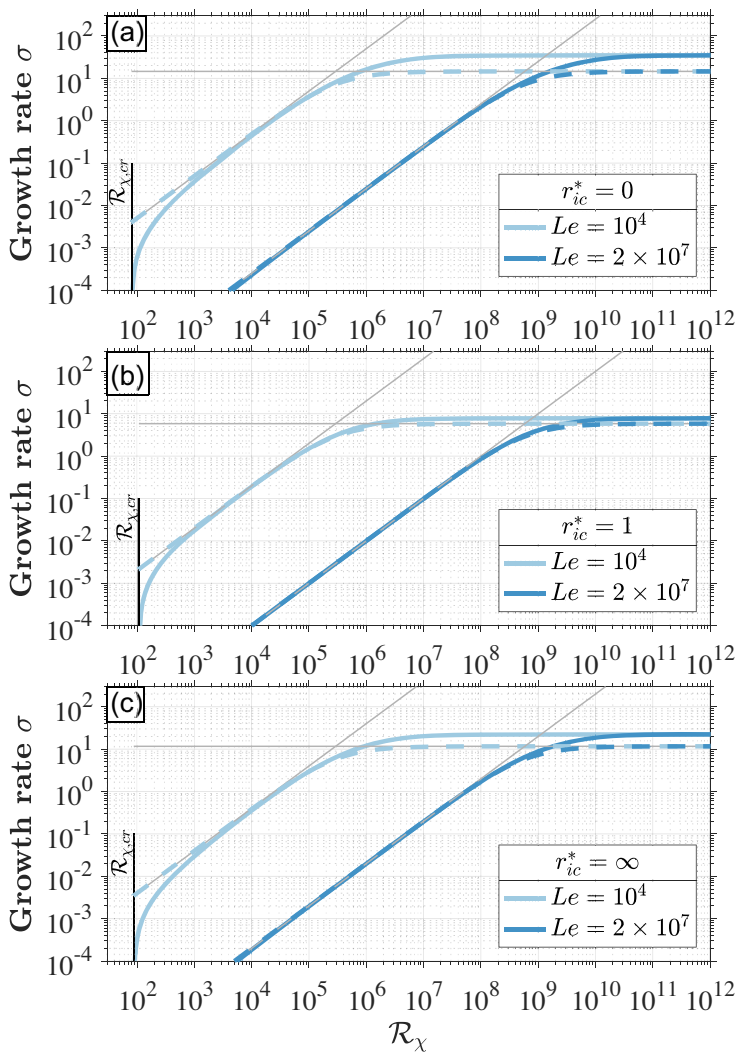

Figure 1. Growth rate of the translation instability as a function of $\mathcal{R}_{\chi}$ at $\frac{\alpha_{T} \Delta \Theta}{\alpha_{c} \Delta \chi}=-3, L e=10^{4}$ or $2 \times 10^{7}$, and (a) $r_{\mathrm{ic}}^{*}=0$, (b) $r_{\mathrm{ic}}^{*}=1$ and (c) $r_{\text {ic }}^{*}=\infty$. The thick solid lines show the numerical solutions of the dispersion equation (A43); the dashed lines show the approximate solution given in eq. (50). The thin grey lines show the two asymptotes given by eq. (51).

(see Appendix A3.3 for the derivation). This has the two following limits:

$\sigma \simeq \frac{\kappa_{T}}{r_{\text {ic }}^{2}} \frac{1 / 3-2 / 5 r_{\text {ic }}^{*}}{2 / 3-r_{\text {ic }}^{*}} \begin{cases}\mathcal{R}_{\chi} / L e & \text { if }-\mathcal{R}_{\Theta} \ll 175 / 2, \\ \frac{175}{2} \frac{\alpha_{c} \Delta \chi}{-\alpha_{T} \Delta \Theta} & \text { if }-\mathcal{R}_{\Theta} \gg 175 / 2 .\end{cases}$

The approximate solution eq. (50) is shown on Fig. 1 with thick dashed lines. The thin grey lines show the two asymptotes given by eq. (51). The two asymptotes intersect at $\mathcal{R}_{\Theta}=-175 / 2$, the corresponding value of $\mathcal{R}_{\chi}$ being given by $\frac{\alpha_{c} \Delta \chi}{\alpha_{T} \Delta \Theta} L e \mathcal{R}_{\Theta}=-\frac{175}{2} \frac{\alpha_{c} \Delta \chi}{\alpha_{T} \Delta \Theta} L e$. The agreement with the full solution (thick solid lines) is excellent for $-\mathcal{R}_{\Theta} \ll 175 / 2$. At $-\mathcal{R}_{\Theta} \gg 175 / 2$, the approximate solution underestimates the actual growth rate, by up to a factor 3 at $r_{\mathrm{ic}}^{*}=0$, but is still rather good at $r_{\text {ic }}^{*}$ around 1 .

Note that since $\mathcal{R}_{\chi} /$ Le $\propto 1 / \kappa_{T}$, the growth rate is independent of the thermal and compositional diffusivities if $-\mathcal{R}_{\Theta} \ll 175 / 2$. On the other hand, the growth of the instability is limited by the diffusion of the temperature heterogeneity when $-\mathcal{R}_{\Theta} \gg 175 / 2$; the growth rate is inversely proportional to the thermal diffusion timescale, but independent of the compositional diffusion timescale.

\section{STEADY-STATE TRANSLATION}

We now derive expressions for the translation rate in steady-state conditions, still focusing on the limit $\mathcal{P} \rightarrow 0$ described by the set of equation of Section 2.3. The radius of the inner core is assumed to be constant, and so are the heat and composition source terms $S_{T}$ and $S_{c}$ in eqs (1) and (3). In the stability analysis of Section 4, the base potential temperature and composition profiles were imposed, and the definition of the Rayleigh numbers were based on the assumed potential temperature and composition differences across the inner core, which were left unspecified. This cannot be done anymore because the potential temperature and composition profiles now depend on the translation rate, and are therefore outputs of the problem. We, therefore, non-dimensionalize the potential temperature and composition by constructing potential temperature and composition scales $\Delta \Theta$ and $\Delta \chi$ from the heat and composition sources terms:

$\Delta \Theta=\frac{S_{T} r_{\mathrm{ic}}^{2}}{6 \kappa_{T}}, \quad \Delta \chi=\frac{S_{c} r_{\mathrm{ic}}^{2}}{6 \kappa_{c}}$,

which correspond to steady-state conductive profiles.

\subsection{Phenomenology}

Fig. 2 shows the translation rate $V$ obtained by solving the set of equations corresponding to the rigid inner core limit [eqs (15), (19) and (20)], for various values of $\mathcal{R}_{\chi}, L e$ and of the buoyancy ratio $\mathcal{B}$ defined as the ratio of the production rates of thermal and compositional buoyancy:

$\mathcal{B}=\frac{\alpha_{T} S_{T}}{\alpha_{c} S_{c}}=\frac{\mathcal{R}_{\Theta}}{\mathcal{R}_{\chi}} L e^{2}=\frac{R a_{\Theta}}{R a_{\chi}} L e^{2}$.

(Note that this ratio is different from the ratio $\frac{\alpha_{T} \Delta \Theta}{\alpha_{c} \Delta x}$ used in Section 4.) Here the compositional field is always assumed to be destabilizing $\left(\mathcal{R}_{\chi}>0\right)$, so $\mathcal{B}>0$ means that both compositional and thermal fields are destabilizing, while $\mathcal{B}<0$ means that the compositional field is destabilizing and the thermal field stabilizing. The steady-state potential temperature and composition fields in crosssections of the inner core are shown in Fig. 3 for three of these calculations (red diamonds denoted a to $\mathbf{c}$ on Fig. 2).

Several different regimes are found depending on the relative importances of advection and diffusion of heat and composition, which are measured, respectively, by the thermal Péclet number $P e_{T}=V r_{\text {ic }} / \kappa_{T}$, and compositional Péclet number $P e_{\chi}=V r_{\text {ic }} / \kappa_{c}=L e P e_{T}$. The translation rate being normalized by $\kappa_{T} / r_{\text {ic }}$, the thermal Péclet number is simply equal to the nondimensional translation rate, and the compositional Péclet number is equal to the Lewis number times the translation rate. Since we focus on the translation regime, the relevant diffusion timescales are those of a degree 1 heterogeneity. The free-decay thermal diffusion timescale of a degree 1 temperature heterogeneity is $r_{\text {ic }}^{2} /\left(\alpha_{11}^{2} \kappa_{T}\right) \simeq r_{\text {ic }}^{2} /\left(20.2 \kappa_{T}\right)$, where $\alpha_{11} \simeq 4.4934$ is the first zero of the degree 1 spherical Bessel function of the first kind. ${ }^{1}$ Thermal (resp. compositional) diffusion would, therefore, be small compared to advection if $P e_{T}$ (resp. $P e_{\chi}$ ) is large compared to 20.2.

If $\mathcal{B}>-1$, the net buoyancy gradient is destabilizing, and the translation mode is found to be very similar to thermally driven translation: the compositional and thermal fields display degree 1 heterogeneity patterns, which are more or less pronounced depending on the value of the Péclet numbers. Fig. 3(a) shows the composition and potential temperature fields in a case where both

\footnotetext{
${ }^{1}$ The free-decay thermal diffusion timescale is obtained by looking for a solution of the heat equation of the form $\Theta_{l}(r, t)=\tilde{\Theta}_{l}(r) P_{l}(\cos \theta) \mathrm{e}^{-\lambda t}$. The solution satisfying the condition $\Theta_{l}\left(r_{\text {ic }}, t\right)=0$ is $\Theta_{l}(r, t)=$ $j_{l}\left(\alpha_{l 1} r / r_{\text {ic }}\right) P_{l}(\cos \theta) \mathrm{e}^{-t /\left(\alpha_{l 1}^{2} \kappa / r_{\text {ic }}^{2}\right)}$, where $j_{l}$ is the spherical function of the first kind of degree $l$ and $\alpha_{l 1}$ its first zero.
} 


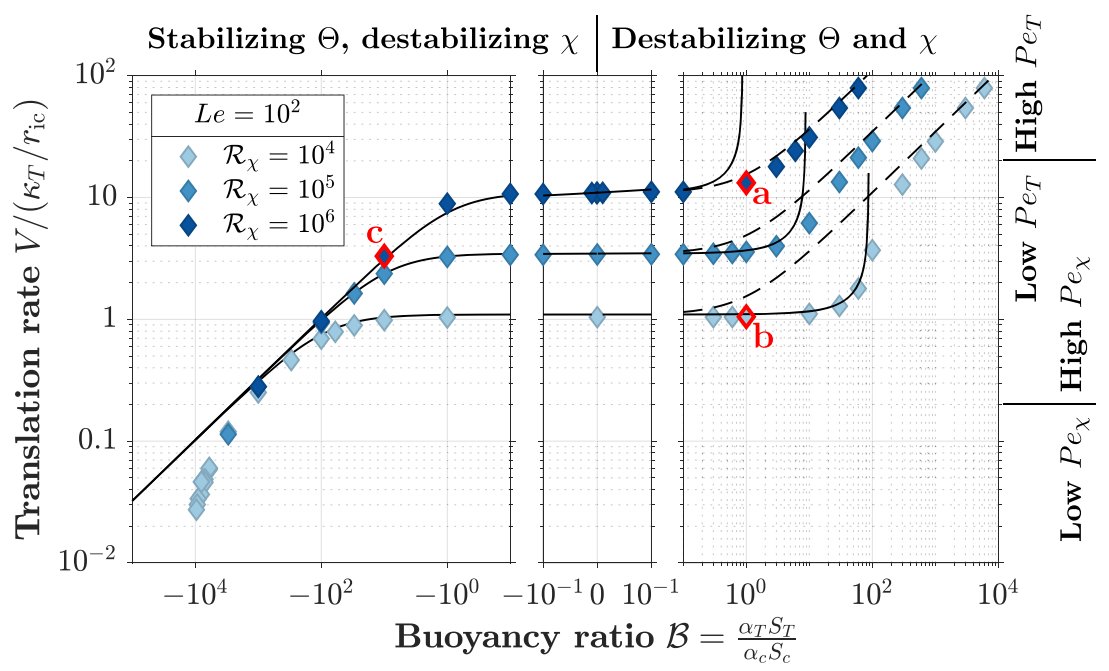

Figure 2. Steady-state translation rate as a function of the buoyancy ratio, Lewis number and compositional Rayleigh number. Filled diamonds show results from the rigid inner core limit numerical model. Black lines are the predictions of the analytical models in the large thermal and compositional Péclet numbers [dashed lines, eq. (57)] and large compositional Péclet number, $\mathcal{O}(1)$ thermal Péclet number [solid lines, eq. (66)] regimes. The red symbols denoted a to $\mathbf{c}$ correspond to the snapshots shown in Fig. 3.

the compositional and thermal Péclet numbers are large compared to 1 . The translation rate is $V=13.2$, which gives $P e_{T}=13.2$ and $P e_{\chi}=1.32 \times 10^{3}$. The composition and potential temperature fields show a degree 1 pattern with higher concentration and potential temperature on the melting side. The composition and potential temperature go back to zero across a boundary layer on the melting side to satisfy the boundary conditions (the compositional boundary layer cannot be seen on the figure, but is resolved in the numerical calculation). The thermal boundary layer is much thicker, owing to the much larger thermal diffusivity (since the boundary layer is where diffusion balances advection, its thickness is on the order of $\left.1 / P e_{T}\right)$. Fig. 3(b) shows the composition and potential temperature fields in a case where the compositional Péclet number is large compared to $1\left(P e_{\chi}=105\right)$, while the thermal Péclet number is on the order of $1\left(P e_{T}=1.05\right)$. The compositional field is similar to the case of Fig. 3(a), though the boundary layer is thicker. In contrast, the potential temperature field is only slightly affected by the translation: it is close to a diffusive field, the isotherms being only slightly shifted towards the melting side of the inner core. An expression for the translation rate in this regime is obtained in Section 5.2.

If $\mathcal{B}<-1$, we find that the translation mode still exists even though the stabilizing thermal density gradient is now larger in magnitude than the destabilizing compositional density gradient, in agreement with the stability analysis of Section 4 . The translation rate-hence the thermal Péclet number-is found to be always smaller than about 10, which means that the Péclet number based on the degree 1 diffusion timescale, $V r_{\text {ic }} /\left(\alpha_{11}^{2} \kappa_{T}\right)$, is always smaller than $\simeq 0.5$. This implies that thermal diffusion is always of importance. This is confirmed by inspection of the potential temperature field pattern. For example, Fig. 3(c) shows the composition and potential temperature fields in a case where $L e=10^{2}, \mathcal{R}_{\chi}=10^{6}$ and $\mathcal{R}_{\Theta}=-10^{3}$, which gives $\mathcal{B}=-10$. The translation rate is $V=3.3$, which gives $P e_{T}=3.3$ and $P e_{\chi}=330$. The compositional field is very similar to the $\mathcal{B}=1$ cases, but the asymmetry of the potential temperature field is much less pronounced because of the effect of diffusion. This is a distinctive feature of the double-diffusive nature of the translation mode: the translation can perdure only if the degree 1 potential temperature heterogeneity diffuses fast enough for the net buoyancy contribution of the thermal field to remain smaller than the compositional contribution. In practice, the volume integrated thermal buoyancy almost balances the compositional buoyancy, but thermal diffusion allows a small imbalance which drives the translation. This is similar to the mechanism of the more classical salt finger regime of double-diffusive convection (e.g. Turner 1974), except that it happens at a much larger length scale. In the classical salt finger regime, convection develops in the form of narrow vertical plumes with a width selected by the instability to allow efficient heat diffusion but negligible compositional diffusion (Stern 1960). This has the effect of reducing the stabilizing effect of thermal buoyancy while letting the compositional buoyancy unaffected, hence promoting convection. In the translation regime, the length scale is fixed at $\sim r_{\text {ic }}$ (i.e. there is only one 'salt finger'), and the translation rate is limited by the necessity to smooth out the lateral temperature heterogeneities by diffusion. In other words, the translation rate is selected to allow for efficient heat diffusion but negligible compositional diffusion.

We derive in the next two subsections analytical expressions for the translation rate obtained under the assumption that both the thermal and compositional Péclet numbers are large (Section 5.2), or that the compositional Péclet number is large and the thermal Péclet number on the order of 1 or smaller (Section 5.3). The $P e_{\chi} \gg 1, P e_{T}$ $\gg 1$ case is not relevant for the double-diffusive regime, but would be relevant for the inner core if its thermal history and thermal diffusivity are such that the temperature field is destabilizing. It is also a direct extension of the model of thermal translation developed in Alboussière et al. (2010) and Deguen et al. (2013), and is therefore useful as a reference. The analytical solutions are shown on Fig. 2 in black dashed lines and black solid lines, respectively. Note that the domain of validity of these solutions are really defined in terms of the Péclet numbers, which depend on the Rayleigh numbers and Lewis number. In particular, the $P e_{\chi} \gg 1, P e_{T}=\mathcal{O}(1)$ solution is valid in the double-diffusive regime $(\mathcal{B}<-1)$ but happens to also correctly predict $V$ for the $\mathcal{B}>-1$ cases when the translation rate is not too large. 


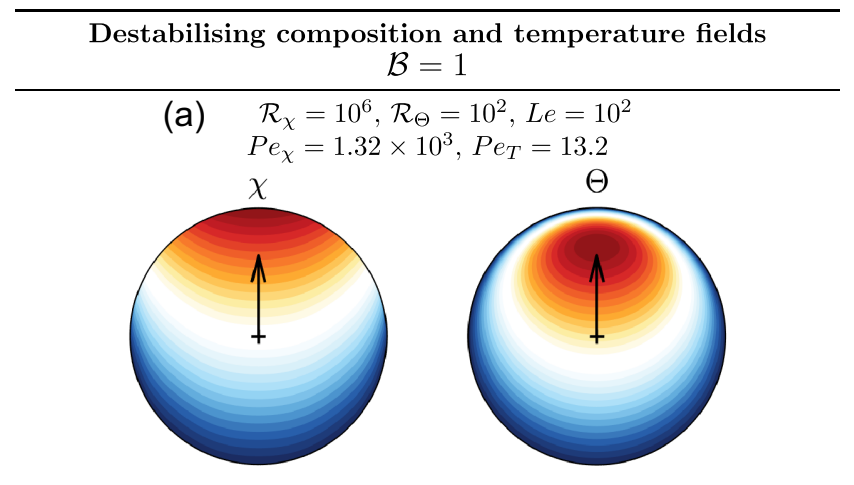

(b) $\quad \mathcal{R}_{\chi}=10^{4}, \mathcal{R}_{\Theta}=1, L e=10^{2}$ $P e_{\chi}=105, P e_{T}=1.05$
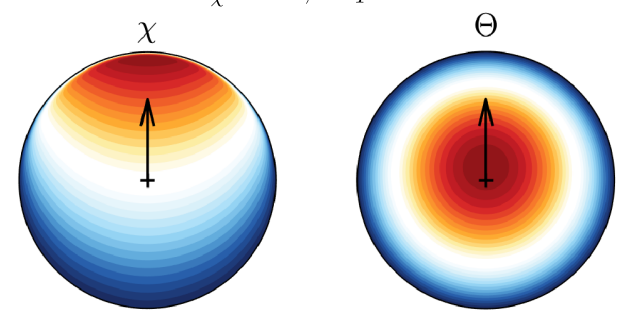

Double diffusive regime :

destabilising composition field, stabilising temperature field $\mathcal{B}=-10$

(c)

$\mathcal{B}=-10$
$\mathcal{R}_{\chi}=10^{6}, \mathcal{R}_{\Theta}=-10^{3}$, Le $=10^{2}$

$P e_{\chi}=3.3 \times 10^{2}, P e_{T}=3.3$
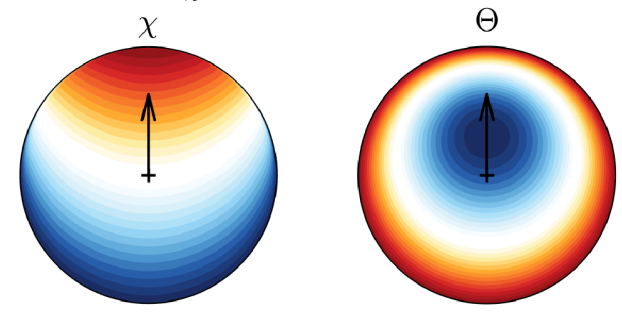

Figure 3. Steady-state composition (left) and potential temperature (right) fields in a cross-section of the inner core including the translation direction, obtained by solving the system of equations corresponding to the rigid inner core limit (Section 2.3). The black arrows show the translation direction. Reds means high potential temperature/composition, and blue means low potential temperature/composition. Panels (a) and (b) show the composition and potential temperature fields in cases where they are both destabilizing. Panel (c) corresponds to a case where the composition field is destabilizing and the potential temperature stabilizing.

\subsection{Large thermal and compositional Péclet numbers}

We assume here that diffusion of heat and composition are negligible compared to advection (large thermal and compositional Péclet numbers), and look for a steady-state solution of the translation equations. The translation rate is given by eq. (15) as a function of the degree 1 components of the potential temperature and compositional fields, which can be obtained by considering eqs (19) and (20) with $l=0$, in which diffusion is neglected and a steady state is assumed:

$0=-V \frac{1}{3}\left(\frac{2}{r}+\frac{\partial}{\partial r}\right) \Theta_{1}+6$,

$0=-V \frac{1}{3}\left(\frac{2}{r}+\frac{\partial}{\partial r}\right) \chi_{1}+6 L e^{-1}$.
The solutions of these two equations are

$\Theta_{1}=\frac{6}{V} r, \quad \chi_{1}=L e^{-1} \frac{6}{V} r$.

Inserting these expressions into eq. (15) and solving for $V$ gives the following expression for the translation rate:

$V=\left[\frac{6}{5}\left(\mathcal{R}_{\Theta}+\frac{\mathcal{R}_{\chi}}{L e^{2}}\right)\right]^{1 / 2}=\left[\frac{6}{5} \frac{\mathcal{R}_{\chi}}{L e^{2}}(1+\mathcal{B})\right]^{1 / 2}$.

This is plotted as black dashed lines in Fig. 2. In dimensional form, this writes

$V=r_{\text {ic }}\left(\frac{\rho}{\Delta \rho} \frac{\alpha_{T} S_{T}+\alpha_{c} S_{c}}{5 \tau_{\phi}}\right)^{1 / 2}$.

The translation rate is independent of the thermal and compositional diffusivities, which is consistent with the assumption of large thermal and compositional Péclet numbers. The $S_{c}=0$ limit of eq. (58) corresponds to the translation rate found by Alboussière et al. (2010) in the case of a thermally driven translation.

The magnitude of the thermal and compositional degree 1 heterogeneities can be obtained from eqs (56) and (57) and are given (in dimensional form) by

$\Theta_{1}=\frac{S_{T} r_{\mathrm{ic}}}{V} \frac{r}{r_{\mathrm{ic}}}=\frac{S_{T} r_{\mathrm{ic}}^{2}}{\kappa_{T}}\left[\frac{6}{5} \frac{\mathcal{R}_{\chi}}{L e^{2}}(1+\mathcal{B})\right]^{-1 / 2} \frac{r}{r_{\mathrm{ic}}}$,
$\chi_{1}=\frac{S_{c} r_{\mathrm{ic}}}{V} \frac{r}{r_{\mathrm{ic}}}=\frac{S_{c} r_{\mathrm{ic}}^{2}}{\kappa_{T}}\left[\frac{6}{5} \frac{\mathcal{R}_{\chi}}{L e^{2}}(1+\mathcal{B})\right]^{-1 / 2} \frac{r}{r_{\mathrm{ic}}}$.

\subsection{Large compositional Péclet number, $\mathcal{O}(1)$ thermal Péclet number}

We now focus on the case of double-diffusive translation, where the potential temperature gradient is stabilizing and larger in magnitude than the destabilizing compositional gradient. In this situation, we can expect the rate of translation to be limited by the diffusion of the degree 1 component of the potential temperature field. While the compositional Péclet number may still be large, the thermal Péclet number is necessarily of order 1 or smaller.

In this limit, we still have

$\chi_{1}=L e^{-1} \frac{6}{V} r$,

but the degree 1 component of the potential temperature field now results from a balance between production of degree 1 through translation of the degree 0 , and diffusion of the degree $1 . \Theta_{1}$ can be obtained by considering eq. (19) with $l=1$, which writes

$0=-V \frac{2}{5}\left(\frac{3}{r}+\frac{\partial}{\partial r}\right) \Theta_{2}-V \frac{\partial \Theta_{0}}{\partial r}+\mathcal{D}_{1} \Theta_{1}$

in steady state. Now since the degree 2 component is produced by the translation of the degree 1 component, which here is assumed to remain small compared to the degree 0 component, the two terms in $\Theta_{2}$ in eq. (62) are expected to be small compared to the term due to translation of the degree 0 . We, therefore, neglect these terms and, recalling that $\Theta_{0}=1-r^{2}$, eq. (62) becomes

$0=2 V r+\mathcal{D}_{1} \Theta_{1}$,

the solution of which [using eq. (44) with $\alpha=1$ and the boundary condition $\Theta_{1}=0$ at $\left.r=1\right]$ being

$\Theta_{1}=\frac{V}{5}\left(r-r^{3}\right)$. 
Inserting this expression for $\Theta_{1}$ and the high compositional Péclet number limit for $\chi_{1}$ into eq. (15) and solving for $V$ gives

$$
\begin{aligned}
& V=\left(\frac{6}{5} \frac{\mathcal{R}_{\chi} / L e^{2}}{1-\frac{2}{175} \mathcal{R}_{\Theta}}\right)^{1 / 2} \\
& =\left[\frac{5}{6}\left(\frac{L e^{2}}{\mathcal{R}_{\chi}}-\frac{2}{175} \mathcal{B}\right)\right]^{-1 / 2} .
\end{aligned}
$$

This is plotted as black solid lines in Fig. 2. The translation rate approaches $\sqrt{-105 / \mathcal{B}}$ if $-\mathcal{R}_{\Theta} \gg 175 / 2$. In this limit, the translation rate is, therefore, independent of the phase change timescale. It is instead limited by the rate of diffusion of the degree 1 thermal heterogeneities. Note that in the double-diffusive regime (i.e. $\mathcal{B}<-1$ ), the translation rate is predicted to be smaller than $\sqrt{105}=10.25$, which is consistent with the observation from the numerical simulations that $V$ is smaller than $\simeq 10$ in this regime.

In dimensional form, the translation rate writes

$V=\frac{\kappa_{T}}{r_{\mathrm{ic}}}\left(-\frac{\alpha_{c} S_{c}}{\alpha_{T} S_{T}} \frac{105}{1-525 \frac{\kappa_{T}^{2} \Delta \rho \tau_{\phi}}{\alpha_{T} \rho S_{T} r_{\mathrm{ic}}^{4}}}\right)^{1 / 2}$.

The translation rate approaches $\left(\kappa_{T} / r_{\text {ic }}\right) \sqrt{-105 \frac{\alpha_{c} S_{c}}{\alpha_{T} S_{T}}}$ if $-\mathcal{R}_{\Theta} \gg$ $175 / 2$. The magnitude of the degree 1 temperature and composition heterogeneities can be obtained from eqs (61), (64) and (66), and are given by

$$
\begin{aligned}
\Theta_{1} & =\frac{1}{30} \frac{S_{T} r_{\mathrm{ic}}^{3}}{\kappa_{T}^{2}} V \\
& =\frac{1}{30} \frac{S_{T} r_{\mathrm{ic}}^{2}}{\kappa_{T}}\left[\frac{105}{-\mathcal{B}\left(1-\frac{87.5}{\mathcal{R}_{\Theta}}\right)}\right]^{1 / 2}\left[\frac{r}{r_{\mathrm{ic}}}-\left(\frac{r}{r_{\mathrm{ic}}}\right)^{3}\right], \\
\chi_{1} & =\frac{S_{c} r_{\mathrm{ic}}}{V} \frac{r}{r_{\mathrm{ic}}}=\frac{S_{c} r_{\mathrm{ic}}^{2}}{\kappa_{T}}\left[\frac{105}{-\mathcal{B}\left(1-\frac{87.5}{\mathcal{R}_{\Theta}}\right)}\right]^{1 / 2} \frac{r}{r_{\mathrm{ic}}} .
\end{aligned}
$$

\subsection{Comparison with numerical calculations}

Fig. 2 shows the translation rate obtained from solving numerically eqs (15)-(20) given in Section 2.3 (ignoring any possible deformation of the inner core), together with the predictions of eqs (57) (black dashed lines) and (66) (solid black lines). The agreement is excellent as long as $\mathcal{R}_{\Theta} / \mathcal{R}_{\Theta, \text { cr }}+\mathcal{R}_{\chi} / \mathcal{R}_{\chi, \text { cr }} \gg 1$. The prediction in the $P e_{C} \gg 1, P e_{T}=\mathcal{O}(1)$ limit remains valid when both the thermal and compositional fields are destabilizing as long as the translation rate is smaller than about 10 . Above that value, the high $\mathrm{Pe}_{C}$, high $\mathrm{Pe}_{T}$ prediction is in very good agreement with the numerical solution.

Also shown in Fig. 2 is the value of $\mathcal{B}$ corresponding to the onset of the instability at $L e=10^{2}$ according to the marginal stability condition, calculated as follow. The numerical calculations correspond to a case with constant $S_{\chi}$ and $S_{\Theta}$, for which the critical modified Rayleigh numbers are both equal to $175 / 2$. The marginal stability condition given by eq. (40) then writes

$\mathcal{R}_{\Theta}+\mathcal{R}_{\chi}=\frac{175}{2}$, as found in Section 4. In terms of $\mathcal{B}$, this writes

$\mathcal{B}=\left(\frac{175 / 2}{\mathcal{R}_{\chi}}-1\right) L e^{2}$.

At $L e=10^{2}$ and $\mathcal{R}_{\chi}=10^{4}$, this gives a critical value for $\mathcal{B}$ equal to $-9.9 \times 10^{3}$. This value, shown with a grey line on Fig. 2, is consistent with the onset of the instability obtained numerically.

\section{DEFORMATION AND CONDITIONS OF EXISTENCE OF THE TRANSLATION MODE}

The advection of heat and composition by the translation mode induces $l=1$ horizontal density variations (Fig. 3) which may drive a large-scale creeping flow from the melting hemisphere to the crystallizing hemisphere. This would redistribute the temperature and concentration perturbations in a way that weakens the magnitude of the driving force of the convection mode. In the purely thermal case, it was shown (Deguen et al. 2013; Mizzon \& Monnereau 2013) that the relative importance of this flow and of the translation is a function of $\mathcal{P}$, with the translation mode collapsing when $\mathcal{P} \gtrsim 30$. In the double-diffusive regime, a similar condition on $\mathcal{P}$ is expected, but the conditions of existence of the translation mode are expected to be more severe because the thermal and compositional buoyancy fields almost balance each other at the global scale, thus leading to a relatively small translation rate, but do not balance at the local scale. This implies that the magnitude of the lateral variations of density - and hence the resulting flow-will be larger, for a given translation rate, than in the purely thermal case.

To quantify the importance of deformation and its effect on the translation mode, we solve numerically the system of equations (introduced in Section 2.2) corresponding to a viscously deforming inner core. Fig. 4 shows a regime diagram constructed from an ensemble of axisymmetric calculations, done at a fixed value of $\mathcal{B}=-3$ and $L e=10^{2}$. At fixed $\mathcal{B}$ and $L e$, the ratio of the thermal and compositional Rayleigh numbers is fixed at $\mathcal{B} L e^{-2}$, so that there are only two independent control parameters, $\mathcal{P}$ and either $R a_{\chi}$ or $R a_{\Theta}$. Though the driver of the convection is the compositional field, the translation mode happens to be limited by the value of $R a_{\Theta}$, as demonstrated later in this section [see the discussion surrounding eq. (73)]. We thus show the regime diagram in terms of both $R a_{\chi}$ (left axis) and $-R a_{\Theta}$ (right axis). In Fig. 4 , the thick black line corresponds to the onset of the instability obtained from the complete linear stability analysis (Section 4.1), while the thin black line corresponds to the onset of the translation mode only (Section 4.2.1). Fig. 5 shows snapshots of the potential temperature (left) and composition (right) fields from some of the numerical calculations used in building the regime diagram. Also shown in Fig. 5 are streamlines of the velocity field (left) and of the velocity field minus its translation component (right). The streamlines on the left and right sides would look identical if the translation component of the flow is small.

Figs 4 and 5 show that the translation mode is dominant at small values of $\mathcal{P}$ and moderate Rayleigh numbers. The $\mathcal{P}=0.1$, $R a_{\chi}=10^{2}, R a_{\Theta}=-3 \times 10^{-2}$ case in Fig. 5 is one example of this regime. The streamlines of the flow (left panel) are very close to straight lines, which is consistent with a translation, and the potential temperature and composition fields are similar to the numerical solutions obtained in Section 5 in the rigid inner core limit (Fig. 3). The flow is not a pure translation, as can be seen from the right panels showing the streamlines of the non-translation component of 


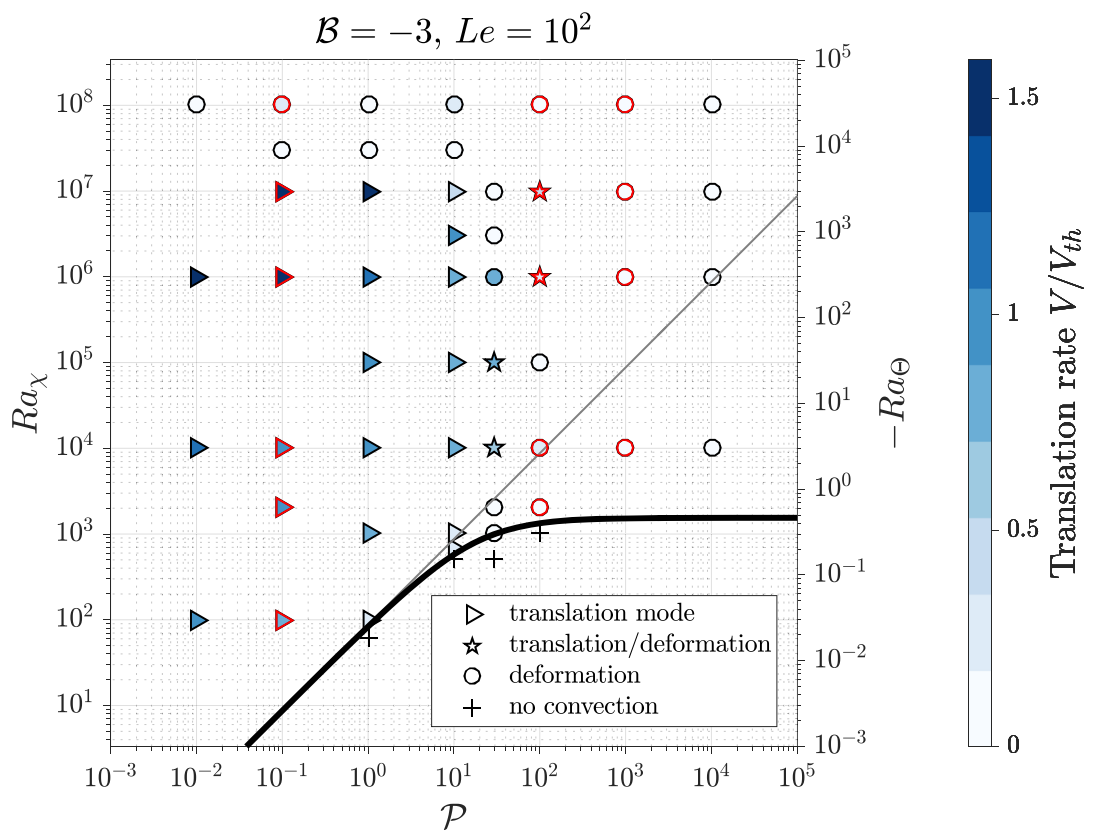

Figure 4. Regime diagram in a $\left(\mathcal{P}, R a_{\Theta}\right)$ space, with $\mathcal{B}=-3$. The colour shading shows the translation rate normalized by the prediction of eq. (66) in the high compositional Péclet, order 1 thermal Péclet regime. $\triangleright$ : calculations in which the translation mode is dominant; $\bigcirc$ : calculations in which the translation component is small compared to the deformation component of the velocity field ; : calculations in which the translation and deformation components of the velocity field are of similar magnitude; +: no convection. The thick black line corresponds to the onset of the instability obtained from the complete linear stability analysis (Section 4.1), while the thin black line corresponds to the onset of the translation mode only (Section 4.2.1). The red symbols correspond to the snapshots shown in Fig. 5.

the flow, which magnitude is small but non-zero (about 1 per cent of the translation rate).

The translation mode appears to be dominant only if $R a_{\chi} \lesssim$ $2 \times 10^{7},-R a_{\Theta} \lesssim 6 \times 10^{3}$ and $\mathcal{P} \lesssim 30$ (Fig. 4 ):

(1) Keeping $\mathcal{P} \lesssim 30$, increasing $R a_{\chi}$ and $-R a_{\Theta}$ results in an increase of the degree 1 component of the potential temperature field, and of the relative magnitude of the non-translation component of the flow: at $\mathcal{P}=0.1, R a_{\chi}=10^{7}, R a_{\Theta}=-3 \times 10^{3}$, it is strong enough to deform the streamlines of the flow (left panel), which now significantly deviate from straight lines (the magnitude of the non-translating component of the velocity field is about 20 per cent of the translation rate). Increasing again the Rayleigh numbers, the translation component of the flow disappears almost totally (see the $\mathcal{P}=0.1, R a_{\chi}=10^{8}, R a_{\Theta}=-3 \times 10^{4}$ case in Fig. 5, where no translation component can be seen from the streamlines pattern).

(2) If $\mathcal{P}$ is increased above $\simeq 30$, the convection pattern just above the onset of convection consists in a degree 1 cell (see e.g. the $\mathcal{P}=$ $10^{3}, R a_{\chi}=10^{4}, R a_{\Theta}=-3$ case in Fig. 5). There is no melting or freezing at the ICB, as can be seen from the fact that the streamlines do not cross the ICB. At higher $R a_{\chi}$, the convection pattern consists in small-scale plumes originating from the boundary layer below the ICB, and becomes more chaotic (see e.g. the $\mathcal{P}=10^{3}, R a_{\chi}=10^{7}$, $R a_{\Theta}=-3 \times 10^{3}$ case in Fig. 5).

The collapse of the translation mode is due to a secondary flow induced by the lateral density variations associated with the potential temperature and composition fields, which redistributes the temperature and composition fields and weakens their degree 1 components, thus decreasing the translation rate. This secondary flow always exists, but its magnitude becomes comparable to the translation rate only if $\mathcal{P}$ or $R a_{\Theta}$ are large enough.

The critical value of $\mathcal{P}$ is consistent with that obtained in the case of thermal convection (Deguen et al. 2013). This transition is due to the fact that, at fixed Rayleigh numbers, increasing $\mathcal{P}$ decreases the translation rate, and therefore increases the relative importance of the secondary flow.

The fact that the translation also collapses when $R a_{\chi}$ and $-R a_{\Theta}$ are large can be understood as follows: Treating the velocity $\mathbf{v}$ of the secondary flow as a small perturbation of the translation, and using the steady-state composition and potential temperature fields of the double-diffusive regime [eqs (61) and (64)], the Stokes equation (5) can be written as

$\nabla^{2} \mathbf{v}=\nabla p-\left[R a_{\Theta} \frac{V}{5}\left(r-r^{3}\right)+\frac{R a_{\chi}}{L e^{2}} \frac{6}{V} r\right] \cos \theta \mathbf{r}$.

We then divide eq. (72) by the translation rate $V$ and replace $V$ in the buoyancy term by the double-diffusive translation rate given by eq. (65). The term proportional to $R a_{\chi} /\left(L e^{2} V^{2}\right)$ gives a term $\propto \mathcal{P}$ plus a term $\propto R a_{\Theta}$ which combines with the term in $R a_{\Theta}$ of eq. (72) to give

$\nabla^{2} \frac{\mathbf{v}}{V}-\frac{1}{V} \nabla p=-\left[R a_{\Theta}\left(\frac{1}{7} r-\frac{1}{5} r^{3}\right)+5 \mathcal{P} r\right] \cos \theta \mathbf{r}$.

It is clear from this equation that $|\mathbf{v}|$ would be a significant fraction of $V$ if either $-R a_{\Theta}$ or $\mathcal{P}$ is large. We thus expect that the redistribution of the thermal and compositional heterogeneities will have a significant effect on the translation rate if either $-R a_{\Theta}$ or $\mathcal{P}$ is large. This is indeed consistent with the results of the numerical calculations. Eq. (73) also suggests that the critical values of $\mathcal{P}$ and $R a_{\Theta}$ should not depend on the value of $\mathcal{B}$ or Le. From Fig. 4, we find that the critical values of $\mathcal{P}$ and $-R a_{\Theta}$ are $\simeq 30$ and $\simeq 6 \times 10^{3}$, respectively.

If both the compositional and thermal fields are destabilizing, inserting into the Stokes equation the large $P e_{C}$, large $P e_{T}$ potential temperature and composition fields [eq. (56)], dividing by $V$ and 


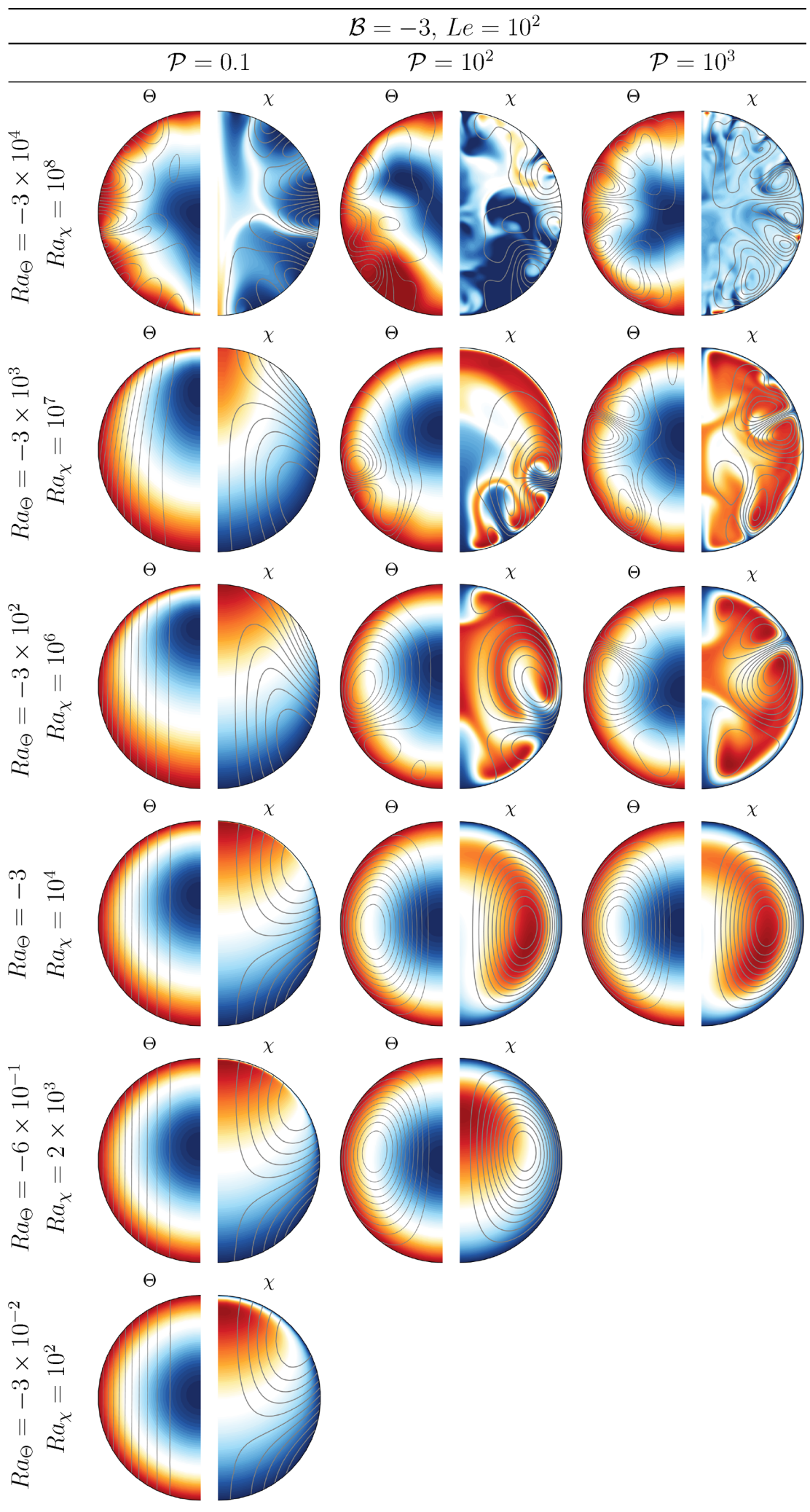

Figure 5. Snapshots of (left) potential temperature (higher values in red, lower values in blue) and streamlines (grey lines) of the flow. Right, light element concentration (higher values in red, lower values in blue) and streamlines (grey lines) of the non-translation component of the flow, for various $\mathcal{P}, R a_{\Theta}, R a_{\chi}$. The snapshots shown here correspond to the numerical solutions denoted by red symbols in the regime diagram shown in Fig. 4. $\mathcal{P}$ increases from left to right, and $R a_{\chi}$ and $-R a_{\Theta}$ increases from bottom to top. $\mathcal{B}$ and $L e$ are kept constant at $\mathcal{B}=-3$ and $L e=10^{2}$. 
taking the translation rate given by (57) yields

$\nabla^{2} \frac{\mathbf{v}}{V}-\frac{1}{V} \nabla p=-5 \mathcal{P} \cos \theta r \mathbf{r}$,

which suggests that in this case, the secondary flow can become a significant fraction of $V$ only if $\mathcal{P}$ is large enough, irrespective of the values of the Rayleigh numbers. This is consistent with the results obtained in the case of a thermally driven translation (Deguen et al. 2013).

\section{APPLICATION TO EARTH'S INNER CORE}

The theoretical and numerical investigations presented in the previous sections show that translation of the inner core in a doublediffusive regime is possible if:

(1) the compositional and thermal fields are such that

$\alpha_{c} \Delta \chi \gtrsim \frac{-\alpha_{T} \Delta \Theta}{L e}$

(Section 4). In practice, the value of Le $\left(2 \times 10^{7}\right)$ and $-\alpha_{T} \Delta \Theta$ are such that even a slightly positive value of $\alpha_{c} \Delta \chi$ is enough to promote double-diffusive translation. However, the growth rate of the instability and the translation rate do depend significantly on the thermal state of the inner core.

(2) the non-dimensional parameters $\mathcal{P}$ and $-R a_{\Theta}$ are smaller than $\simeq 30$ and $\simeq 6 \times 10^{3}$, respectively (Section 6 ). These conditions can be rewritten as a condition for the inner core viscosity, which must be larger than

$\eta_{c}=\max \left(\frac{\Delta \rho g^{\prime} r_{\mathrm{ic}}^{2} \tau_{\phi}}{30},-\frac{\alpha_{T} \rho g^{\prime} S_{T} r_{\mathrm{ic}}^{6}}{3.610^{4} \kappa_{T}^{2}}\right)$

for the translation mode to be dominant. The critical viscosity $\eta_{c}$ is a strongly increasing function of $r_{\text {ic }}$, which means that this condition is much easier to meet early in the inner core history.

\subsection{Thermal evolution of the inner core}

The thermal source term $S_{T}$ can be written as

$S_{T}=\frac{\rho g^{\prime} \gamma T}{2 K_{S}}\left[\left(\frac{\mathrm{d} T_{s}}{\mathrm{~d} T_{\mathrm{ad}}}-1\right) \frac{\mathrm{d} r_{\mathrm{ic}}^{2}}{\mathrm{~d} t}-6 \kappa_{T}\right]$,

where $\mathrm{d} T_{s} / \mathrm{d} T_{\text {ad }} \simeq 1.65$ is the ratio of the Clapeyron slope to the adiabat, $g^{\prime}=\mathrm{d} g / \mathrm{d} r=3.6 \times 10^{-6} \mathrm{~s}^{-2}, \gamma \simeq 1.4$ is the Gruneisen parameter, $K_{S}=1400 \mathrm{GPa}$ the isentropic bulk modulus, $T \simeq 5500 \mathrm{~K}$ the inner core temperature (Deguen \& Cardin 2011). For simplicity, instead of solving the core energy balance to obtain the evolution of $r_{\mathrm{ic}}(t)$, as was done in Deguen et al. (2013), we simply assume an evolution of the form $r_{\mathrm{ic}}=r_{\mathrm{ic}}^{p}\left(t / \tau_{\mathrm{ic}}\right)^{a}$, where $r_{\mathrm{ic}}^{p}=1221 \mathrm{~km}$ is the present-day radius of the inner core and $\tau_{\text {ic }}$ is the age of the inner core. Labrosse (2015) found that this expression with $a$ around 0.43 correctly approximates the exact solution of the core energy balance. The advantage of using the simple parametrization is that an explicit form is obtained for $S_{T}$, with $\mathrm{d} r_{\text {ic }}^{2} / \mathrm{d} t=2 a\left(r_{\text {ic }}^{p}\right)^{2} t^{2 a-1} / \tau_{\text {ic }}^{2 a}$, which makes its evaluation straightforward. For example, with $\kappa_{T}=2 \times 10^{-5}$ $\mathrm{m}^{2} \mathrm{~s}^{-1}$ (corresponding to a thermal conductivity $\simeq 200 \mathrm{~W} \mathrm{~m}^{-1} \mathrm{~K}^{-1}$ (Pozzo et al. 2014)) and $\tau_{\text {ic }}$ taken in the range 0.5-1 Gy, we obtain $S_{T}$ in the range $-1.2 \times 10^{-14} \mathrm{~K} \mathrm{~s}^{-1}$ to $-8 \times 10^{-15} \mathrm{~K} \mathrm{~s}^{-1}$. With $\kappa_{T}=10^{-5} \mathrm{~m}^{2} \mathrm{~s}^{-1}$ (a thermal conductivity $\simeq 100 \mathrm{~W} \mathrm{~m}^{-1} \mathrm{~K}^{-1}$ ), this range becomes $-4 \times 10^{-15} \mathrm{~K} \mathrm{~s}^{-1}<S_{T}<-9 \times 10^{-16} \mathrm{~K} \mathrm{~s}^{-1}$. With a diffusivity equal to $\kappa_{T}=5 \times 10^{-6} \mathrm{~m}^{2} \mathrm{~s}^{-1}$ corresponding to a thermal conductivity $\simeq 50 \mathrm{~W} \mathrm{~m}^{-1} \mathrm{~K}^{-1}, S_{T}$ is positive (i.e. the temperature field is destabilizing) if $\tau_{\text {ic }}<0.88 \mathrm{~Gy}$.

\subsection{Compositional evolution of the inner core}

A convenient starting point for discussing the compositional state of the inner core is the logarithmic derivative of the relation $k=c_{s} / c_{l}$ expressing thermodynamic equilibrium at the ICB:

$\frac{\mathrm{d} c_{s}}{c_{s}}=\frac{\mathrm{d} c_{l}}{c_{l}}+\frac{\mathrm{d} k}{k}$.

The development of an unstable compositional profile in the inner core requires that $\mathrm{d} c_{s} / c_{s}<0$, which depends on both the evolution of the outer core concentration and on the evolution of the partitioning behaviour.

(1) In the standard model of a well-mixed outer core, light elements are preferentially partitioned to the outer core $(k<1)$ and the concentration of these elements builds up in the liquid as the inner core crystallizes. $\mathrm{d} c_{l} / c_{l}$ is therefore positive. The concentration profile in the inner core can then be destabilizing only if $\mathrm{d} k / k$ is negative and larger in magnitude than $\mathrm{d} c_{l} / c_{l}$. According to ab initio calculations of the partitioning behaviour of oxygen and sulphur (Gubbins et al. 2013), the partition coefficients of these elements happen to decrease with decreasing temperature, implying that $\mathrm{d} k / k$ is indeed negative. The relative importance of $\mathrm{d} c_{l} / c_{l}$ and $\mathrm{d} k / k$ then depends on the core composition and radius of the inner core (through ICB temperature). Labrosse (2014) showed that the concentration of a given element at crystallization is well approximated by

$c_{s}^{\mathrm{icb}}=c_{s, 0}^{\mathrm{icb}}\left(1+A r_{\mathrm{ic}}^{3}-B r_{\mathrm{ic}}^{2}\right)$,

where $A$ and $B$ are parameters which depend on the element considered and its abundance, and $c_{s, 0}^{\text {icb }}$ is the concentration in the inner core at nucleation. The $r_{\mathrm{ic}}^{3}$ term is associated with the compositional evolution of the outer core, and promotes the formation of a stable compositional profile. The $r_{\text {ic }}^{2}$ term is due to the temperature dependence of the partition coefficient, and promotes the formation of an unstable compositional profile. Labrosse (2014) found that, for plausible core composition models, oxygen has always a destabilizing contribution while sulfur has a destabilizing contribution only while the inner core radius is smaller than $\simeq 500 \mathrm{~km}$. The net compositional profile becomes stabilizing when the radius of the inner core reaches $600-900 \mathrm{~km}$ (depending on the composition of the core). Double-diffusive translation could thus plausibly set in early in the inner core history, but would be unlikely to last until today.

From eq. (79), we find that the composition source term is

$S_{c}=-\frac{\mathrm{d} c_{s}^{\mathrm{icb}}}{\mathrm{d} t}=-c_{s, 0}^{\mathrm{icb}}\left(A \frac{\mathrm{d} r_{\mathrm{ic}}^{3}}{\mathrm{~d} t}-B \frac{\mathrm{d} r_{\mathrm{ic}}^{2}}{\mathrm{~d} t}\right)$

which we will evaluate for different scenarios in Section 7.4, and use to obtain the translation rate when relevant.

(2) The situation would be more favourable to convection if the light elements concentration of the liquid from which the inner core crystallizes increases at a slower pace than what is predicted under the assumption of perfect mixing of the outer core, or even decreases with time. This may happen for at least three reasons:

(i) If light elements expelled from the inner core do not mix perfectly in the outer core and accumulate in a stably stratified 


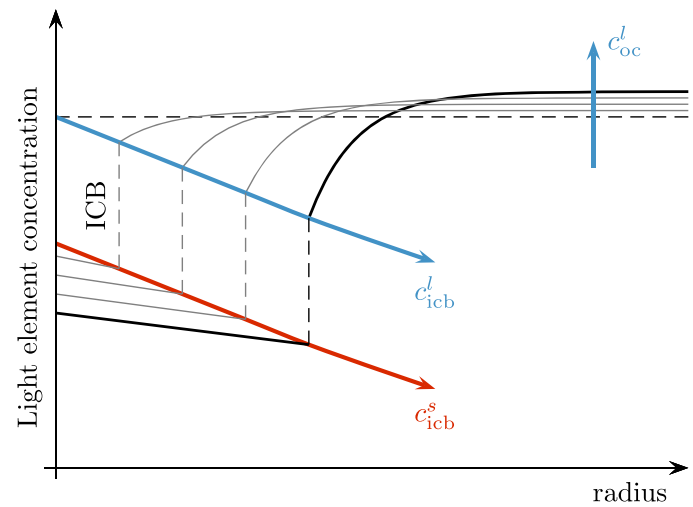

Figure 6. A schematic showing qualitatively the expected effect on the inner core composition of the formation of an iron-rich layer above the ICB. The thin black lines correspond to concentration profiles in the core at different times, with the thick black line corresponding to the current state of the core. The blue and red curves show the evolution of the concentration at the ICB in the solid and liquid phases, respectively. The concentration in the bulk of the outer core increases in response of inner core crystallization, but the concentration at the ICB decreases.

layer below the CMB (e.g. Fearn \& Loper 1981; Braginsky 2006; Bouffard et al. 2017), the light elements concentration just above the ICB would increase at a slower rate than in the perfect mixing model. The exact evolution of the composition of the bulk of the outer core would depend on the mixing efficiency.

(ii) Core-mantle chemical interactions can buffer the core composition, or even induce a decrease of the concentration of some light elements. In particular, exsolution of $\mathrm{MgO}$ or $\mathrm{SiO}_{2}$ (Badro et al. 2016; O'Rourke \& Stevenson 2016; Hirose et al. 2017; O'Rourke et al. 2017) would make the concentration of $\mathrm{O}$ and Si progressively decrease as the core cools down.

(iii) Finally, the existence of the F-layer argues for a secular decrease of light elements concentration close to the ICB, if its interpretation as a stably stratified, iron-rich layer formed as a result of inner core growth is correct. Unless the F-layer is of primordial origin (Arkani-Hamed 2017; Jacobson et al. 2017), its formation implies that the inner core has been crystallizing from an increasingly iron-rich melt (Fig. 6), irrespectively of its formation mechanism (Gubbins et al. 2008; Alboussière et al. 2010; Gubbins et al. 2011). In this situation, both $\mathrm{d} c_{l} / c_{l}$ and $\mathrm{d} k / k$ are negative and the composition field is always destabilizing.

\subsection{Growth rate of the translation instability}

Though instability appears almost unavoidable when the compositional profile is destabilizing, whether the translation rate eventually becomes significant depends on the timescale of instability growth (the inverse of the growth rate $\sigma$ ) compared to the duration of the unstable phase, and on the magnitude of the initial perturbations. From eq. (51), the instability growth rate $\sigma$ is at most 10 to 30 times $\kappa_{T} / r_{\text {ic }}^{2}$ (depending on $r_{\text {ic }}^{*}$ ), which corresponds to a timescale of instability growth $1 / \sigma$ between $\sim 80$ and $240 \mathrm{Myr}$. This is smaller than the age of the inner core, but this does not ensure by itself a significant translation rate. The time needed to reach a given translation rate depends on the magnitude of the initial perturbation, with $1 / \sigma$ being the time needed to increase the translation rate by a factor $e$ $\simeq 2.72$. The translation rate increases by a factor of 10 in a time $(\ln 10) / \sigma \simeq 2.3 / \sigma$, which is in the range 180-550 Myr. This is of the same order of magnitude than the inner core age, which implies that a relatively strong perturbation is needed to ensure a significant translation rate is reached. One possible candidate is heterogeneous growth of the inner core (Sumita \& Olson 1999; Aubert et al. 2008; Aubert 2013; Driscoll 2015). For example, if convection in the outer core imposes a growth pattern with a persisting degree 1 anomaly of amplitude one tenth of the mean solidification rate, it will take about $200 \mathrm{Myr}$ for the translation rate to reach a value similar to the mean inner core growth.

\subsection{Possible scenarios}

Fig. 7 shows the results of calculations corresponding to two different scenarios for the compositional evolution of the core, as well as two different thermal histories (parametrized by inner core age). The thermal diffusivity is fixed at $\kappa_{T}=2 \times 10^{-5} \mathrm{~m}^{2} \mathrm{~s}^{-1}$ and the radius of the inner core is assumed to grow as $r_{\mathrm{ic}}=r_{\mathrm{ic}}^{p}\left(t / \tau_{\mathrm{ic}}\right)^{0.43}$, with $\tau_{\text {ic }}=0.5$ or $1 \mathrm{~Gy}$. The core is assumed to contain 2.42 per cent $\mathrm{O}$ and 6.3 per cent $\mathrm{S}$, following Gubbins et al. (2013) and Labrosse (2014). The buoyancy source terms $\alpha_{c} S_{c}$ and $\alpha_{T} S_{T}$ are calculated from eqs (80) and (77) (Figs 7a and d). This is then used to calculate the non-dimensional numbers $\mathcal{B}, \mathcal{R}_{\chi}$ and $\mathcal{R}_{\Theta}$, from which we obtain the theoretical steady-state translation rate from eqs (57) and (66) (Figs $7 \mathrm{~b}$ and e). In addition, we compute the translation rate by solving numerically the 'rigid inner core' set of equations (Section 2.3), taking into account the growth of the inner core and its thermal and compositional evolution. The instability is initiated numerically by imposing a small initial degree 1 growth heterogeneity $V_{\text {in }}$ of either $10^{-12}$ or $10^{-11} \mathrm{~m} \mathrm{~s}^{-1}$. Finally, we show in Figs $7(\mathrm{c})$ and (f) the critical viscosity $\eta_{c}$ above which the translation mode is dominant. According to eq. (76), $\eta_{c}$ does not depend on the composition field; its evolution is therefore the same in the two scenarios of compositional evolution.

\subsubsection{Crystallization from a well-mixed outer core}

Fig. 7 (left panel) shows the results of calculations made under the assumption that the outer core is well-mixed.

For the two thermal histories considered here, $\alpha_{T} S_{T}$ is negative, except in the very beginning of inner core crystallization. $\alpha_{c} S_{c}$ starts positive, but becomes negative when the inner core radius exceeds $\simeq 600 \mathrm{~km}$. As a result, translation is only possible while $r_{\text {ic }}<600 \mathrm{~km}$.

The translation rate obtained from the steady-state expressions is typically similar to the inner core growth rate. However, this is misleading because the duration of the unstable phase is rather short compared with the timescale of instability growth. Depending on the thermal history considered, it takes between 100 and $200 \mathrm{Myr}$ for the inner core to reach a radius of $600 \mathrm{~km}$. Since the timescale of instability growth is larger than $200 \mathrm{Myr}$, this implies that the steady state regime is unlikely to be reached. This is confirmed by the results of the numerical solution of the 'rigid inner core' set of equations, which shows that in effect the inner core does not reach an appreciable translation rate before becoming stable against the double-diffusive instability. For example, starting with a forced translation with a translation rate of $10^{-11} \mathrm{~m} \mathrm{~s}^{-1}$ with an inner core nucleating 0.5 Gy ago, the translation rate increases by a factor of merely 1.5 before decreasing quickly. If the inner core started crystallizing $1 \mathrm{~Gy}$ ago, the translation rate reaches a maximum only 10 per cent higher than the initial perturbation. We therefore conclude that a significant translation is unlikely in this scenario. 

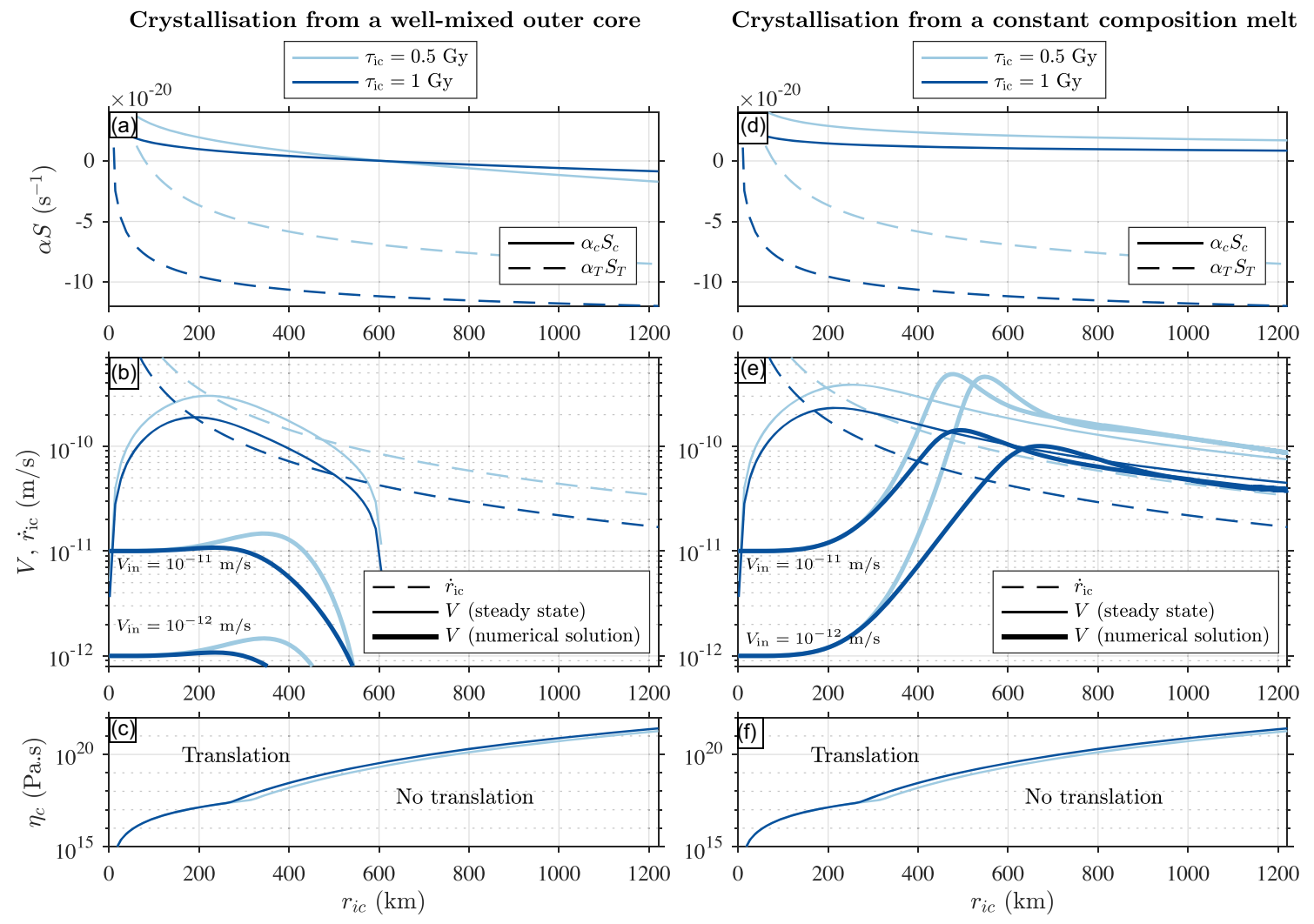

Figure 7. Left column: results of calculations corresponding to a scenario in which the inner core is assumed to crystallize from a well-mixed outer core, the composition of the crystallizing inner core being given by eq. (79). Two thermal histories are considered, with the inner core starting to crystallize either 1 Gy ago (dark blue lines) or 0.5 Gy ago (light blue lines). (a) Solid lines: compositional buoyancy source $\alpha_{c} S_{c}$ obtained from eq. (80); dashed lines: thermal buoyancy source $\alpha_{T} S_{T}$ obtained from eq. (77). (b) Dashed lines: inner core growth rate $\dot{r}_{\text {ic }}$; thin solid lines: translation rate obtained from the steady-state solutions [eqs (57) and (66)]; heavy solid lines: translation rate obtained by solving numerically the 'rigid inner core' set of equations (Section 2.3), with an imposed degree 1 growth heterogeneity $V_{\text {in }}$ of either $10^{-11} \mathrm{~m} \mathrm{~s}^{-1}$ or $10^{-12} \mathrm{~m} \mathrm{~s}^{-1}$. (c) Critical viscosity above which the translation regime dominates [eq. (76)]. Right column: same as panels (a)-(c) if the inner core is assumed to crystallize from a melt of constant composition. The composition of the crystallizing inner core is given by eq. (79) with $A=0$.

\subsubsection{Crystallization from a constant composition melt}

Fig. 7 (right panel) shows the results of calculations made under the assumption that the inner core crystallizes from a melt of constant composition, which amounts to taking $A=0$ in eq. (80).

The thermal histories are the same as in the previous section, $\alpha_{T} S_{T}$ being negative for most of the inner core history. However, $\alpha_{c} S_{c}$ is now always positive and the inner core is always unstable against the double-diffusive translation instability. The translation rate obtained from the steady-state expressions is typically about two times higher than the inner core growth rate. The translation rate obtained from solving the 'rigid inner core' set of equations approaches the steadystate theory after a phase of instability growth, whose duration depends on the magnitude of the initial perturbation. The slight difference between the steady-state theory and the numerical results can be ascribed to the time variation of the inner core radius and source terms.

The critical viscosity $\eta_{c}$ above which translation is possible is a strong function of inner core radius. For example, at $r_{\mathrm{ic}}=200 \mathrm{~km}$, the translation mode is preferred if the inner core viscosity is larger than $\simeq 10^{17} \mathrm{~Pa}$ s. The critical viscosity strongly increases with radius, and reaches about $2-3 \times 10^{21} \mathrm{~Pa}$ s at the current inner-core radius of $1221 \mathrm{~km}$. This does not depend on the composition evolution, and only slightly on the thermal evolution. The final critical viscosity is clearly on the high end part of the range of published estimates, and marginally within the $10^{20}-10^{22} \mathrm{~Pa}$ s range predicted by Reaman et al. $(2011,2012)$. If the viscosity is in the range of $10^{16}$ to $3 \times 10^{21}$ $\mathrm{Pa}$ s, the inner core may have been in the translation regime during a possibly significant part of its history, before leaving the translation field when the critical viscosity becomes higher than the inner core viscosity.

\section{CONCLUSION}

We have demonstrated that double-diffusive translation of Earth's inner core is possible if two conditions are met: (i) the compositional profile within the inner core is destabilizing (almost irrespectively of the thermal state); and (ii) the inner core viscosity is sufficiently large, the required value being a strongly increasing function of the inner core size. The first condition was likely met when the inner core was smaller than about half its present size, and possibly still is if the outer core is imperfectly mixed and has developed stratified regions either below the CMB or above the ICB (Gubbins et al. 2013; Labrosse 2014). The second condition is possibly met for a large part of inner core's history according to the viscosity estimate of Reaman et al. $(2011,2012)$, but would require the inner core viscosity to be larger than $3 \times 10^{21} \mathrm{~Pa}$ s to be satisfied today (see Fig. 7).

If these conditions are satisfied, the effective development of the translation instability further requires that: (i) the duration of the 
unstable phase is long enough compared to the timescale of instability growth, which can be in the range 200-500 Myr depending on the shape of the compositional profile (see Sections 4.2.2 and 7.3); and (ii) that a perturbation of sufficient magnitude is imposed on the inner core and persists on a timescale similar to the instability growth timescale. The first condition makes the development of double-diffusive translation unlikely in the classical model of compositional evolution of the outer core assuming perfect mixing. However, imperfect mixing of the outer core lengthens the duration of the compositionally unstable phase, potentially enough to allow the development of the translation instability. If the inner core crystallizes from a constant composition melt (Section 7.4.2 and Fig. 7), the compositional profile remains destabilizing up to its current radius. The formation of a stably stratified iron-rich layer above the inner core, as the seismologically observed F-layer could be interpreted, would even increase the destabilizing effect of composition.

In scenarios where the inner core is indeed translating, one interesting feature of the double-diffusive regime is that the translation rate depends predominantly on the thermal diffusivity and buoyancy ratio, and only weakly on the melting/solidification timescale, whose value is more uncertain (Buffett \& Matsui 2015). With the thermal and compositional evolution scenarios considered here, the translation rate is found to be typically similar or slightly higher than the inner core growth rate. This is about an order magnitude smaller than what was predicted for a purely thermally driven translation (Alboussière et al. 2010; Deguen et al. 2013; Mizzon \& Monnereau 2013). This happens to be in better agreement with the geodynamo calculations of Aubert et al. (2013) and Aubert (2013), which best reproduce the morphology of the Earth's magnetic field with a degree 1 buoyancy flux heterogeneity at the ICB corresponding to a translation rate slightly smaller than the inner core growth rate.

\section{ACKNOWLEDGEMENTS}

We thank Mathieu Dumberry, an anonymous reviewer, and the editor Richard Holme for their helpful comments on the paper.

\section{REFERENCES}

Abramovich, M. \& Stegun, I., 1965. Handbook of Mathematical Functions, Fourth Printing, Applied Math. Ser. 55, US Government Printing Office.

Alboussière, T., Deguen, R. \& Melzani, M., 2010. Melting induced stratification above the Earth's inner core due to convective translation, Nature, 466, 744-747.

Arkani-Hamed, J., 2017. Formation of a solid inner core during the accretion of Earth, J. geophys. Res., 122(5), 3248-3285.

Aubert, J., 2013. Flow throughout the Earth's core inverted from geomagnetic observations and numerical dynamo models, Geophys. J. Int., 192(2), 537-556.

Aubert, J., Amit, H., Hulot, G. \& Olson, P., 2008. Thermochemical flows couple the Earth's inner core growth to mantle heterogeneity, Nature, 454(7205), 758-761.

Aubert, J., Finlay, C.C. \& Fournier, A, 2013. Bottom-up control of geomagnetic secular variation by the earth's inner core, Nature, 502(7470), 219.

Badro, J., Siebert, J. \& Nimmo, F., 2016. An early geodynamo driven by exsolution of mantle components from Earth's core, Nature, 536(7616), 326-328.

Bergman, M., Lewis, D., Myint, I., Slivka, L., Karato, S. \& Abreu, A, 2010. Grain growth and loss of texture during annealing of alloys, and the translation of Earth's inner core, Geophys. Res. Lett., 37(22), L22313.
Bouffard, M., Labrosse, S., Choblet, G., Fournier, A, Aubert, J. \& Tackley, P.J., 2017. A particle-in-cell method for studying double-diffusive convection in the liquid layers of planetary interiors, J. Comput. Phys., 346, 552-571

Braginsky, S.I., 2006. Formation of the stratified ocean of the core, Earth planet. Sci. Lett., 243, 650-656.

Buffett, B.A., 1997. Geodynamics estimates of the viscosity of the Earth's inner core, Nature, 388, 571-573.

Buffett, B.A. \& Matsui, H., 2015. The fluid dynamics of inner-core growth, Phys. Earth planet. Inter., 243, 22-29.

Calvet, M. \& Margerin, L., 2018. Shape preferred orientation of iron grains compatible with Earth's uppermost inner core hemisphericity, Earth planet. Sci. Lett., 481, 395-403.

Chandrasekhar, S., 1961. Hydrodynamic and Hydromagnetic Stability, International Series of Monographs on Physics, Clarendon.

Cormier, V.F., Attanayake, J. \& He, K., 2011. Inner core freezing and melting: constraints from seismic body waves, Phys. Earth planet. Inter., 188, 163 172.

Davies, C., Silva, L. \& Mound, J., 2013. On the influence of a translating inner core in models of outer core convection, Phys. Earth planet. Inter, 214, 104-114.

Deguen, R., Alboussière, T. \& Cardin, P., 2013. Thermal convection in Earth's inner core with phase change at its boundary, Geophys. J. Int., 194(3), 1310-1334.

Deguen, R. \& Cardin, P., 2011. Thermo-chemical convection in Earth's inner core, Geophys. J. Int., 187, 1101-1118.

de Koker, N., Steinle-Neumann, G. \& Vlcek, V., 2012. Electrical resistivity and thermal conductivity of liquid Fe alloys at high $\mathrm{P}$ and $\mathrm{T}$, and heat flux in Earth's core, Proc. Natl. Acad. Sci. USA, 109(11), 4070-4073.

Deuss, A, Irving, J. \& Woodhouse, J., 2010. Regional variation of inner core anisotropy from seismic normal mode observations, Science, 328(5981), 1018-1020.

Driscoll, P.E., 2015. Testing the dynamic coupling of the core-mantle and inner core boundaries, J. geophys. Res., 120(7), 4689-4701.

Dziewonski, A.M. \& Anderson, D.L., 1981. Preliminary reference Earth model, Phys. Earth planet. Inter, 25, 297-356.

Fearn, D.R. \& Loper, D.E., 1981. Compositional convection and stratification of Earth's core, Nature, 289, 393-394.

Garcia, R., 2002. Constraints on upper inner-core structure from waveform inversion of core phases, Geophys. J. Int., 150, 651-664.

Geballe, Z., Lasbleis, M., Cormier, V. \& Day, E., 2013. Sharp hemisphere boundaries in a translating inner core, Geophys. Res. Lett., 40(9), 17191723.

Gillet, N., Pais, M.A. \& Jault, D., 2009. Ensemble inversion of timedependent core flow models, Geochem. Geophys. Geosyst., 10, Q06004, doi:10.1029/2008GC002290.

Gomi, H., Hirose, K., Akai, H. \& Fei, Y., 2016. Electrical resistivity of substitutionally disordered hcp $\mathrm{Fe}-\mathrm{Si}$ and $\mathrm{Fe}-\mathrm{Ni}$ alloys: Chemically-induced resistivity saturation in the Earth's core, Earth planet. Sci. Lett., 451, 51-61.

Gomi, H., Ohta, K., Hirose, K., Labrosse, S., Caracas, R., Verstraete, M.J. \& Hernlund, J.W., 2013. The high conductivity of iron and thermal evolution of the earth's core, Phys. Earth planet. Inter., 224, 88-103.

Gubbins, D., Alfè, D. \& Davies, C., 2013. Compositional instability of Earth's solid inner core, Geophys. Res. Lett., 40, 1-5.

Gubbins, D., Masters, G. \& Nimmo, F., 2008. A thermochemical boundary layer at the base of Earth's outer core and independent estimate of core heat flux, Geophys. J. Int., 174, 1007-1018.

Gubbins, D., Sreenivasan, B., Mound, J. \& Rost, S., 2011. Melting of the Earth's inner core, Nature, 473(7347), 361-363.

Hirose, K., Morard, G., Sinmyo, R., Umemoto, K., Hernlund, J., Helffrich, G. \& Labrosse, S., 2017. Crystallization of silicon dioxide and compositional evolution of the Earth's core, Nature, 543(7643), 99-102.

Huppert, H.E. \& Turner, J.S., 1981. Double-diffusive convection, J. Fluid Mech., 106, 299-329.

Irving, J., Deuss, A \& Woodhouse, J., 2009. Normal mode coupling due to hemispherical anisotropic structure in Earth's inner core, Geophys. J. Int., 178(2), 962-975. 
Jacobson, S.A., Rubie, D.C., Hernlund, J., Morbidelli, A \& Nakajima, M., 2017. Formation, stratification, and mixing of the cores of Earth and Venus, Earth planet. Sci. Lett., 174, 375-386.

Konôpková, Z., McWilliams, R.S., Gómez-Pérez, N. \& Goncharov, A.F., 2016. Direct measurement of thermal conductivity in solid iron at planetary core conditions, Nature, 534(7605), 99-101.

Koot, L. \& Dumberry, M., 2011. Viscosity of the earth's inner core: constraints from nutation observations, Earth planet. Sci. Lett., 308, 343-349.

Labrosse, S., 2014. Thermal and compositional stratification of the inner core, C. R. Geosci., 346, 119-129.

Labrosse, S., 2015. Thermal evolution of the core with a high thermal conductivity, Phys. Earth planet. Inter., 247, 36-55.

Lasbleis, M., Deguen, R., Cardin, P. \& Labrosse, S., 2015. Earth's inner core dynamics induced by the Lorentz force, Geophys. J. Int., 202, 548-563.

Lasbleis, M., Waszek, L. \& Day, E., 2017. GrowYourIC: a step towards a coherent model of the Earth's inner core seismic structure, Geochem. Geophys. Geosyst., 18, 4016-4026

Lythgoe, K., Deuss, A, Rudge, J. \& Neufeld, J., 2014. Earths inner core: innermost inner core or hemispherical variations?, Earth planet. Sci. Lett., 385, 181-189.

Lythgoe, K.H., Rudge, J.F., Neufeld, J.A. \& Deuss, A, 2015. The feasibility of thermal and compositional convection in Earth's inner core, Geophys. J. Int., 201(2), 764-782.

Mizzon, H. \& Monnereau, M., 2013. Implication of the lopsided growth for the viscosity of Earth's inner core, Earth planet. Sci. Lett., 361, 391-401.

Monnereau, M., Calvet, M., Margerin, L. \& Souriau, A, 2010. Lopsided growth of Earth's inner core, Science, 328, 1014-1017.

Morelli, A, Dziewonski, A.M. \& Woodhouse, J.H., 1986. Anisotropy of the inner core inferred from PKIKP travel times., Geophys. Res. Lett., 13, $1545-1548$.

Mound, J.E. \& Buffett, B.A., 2006. Detection of a gravitational oscillation in length-of-day, Earth planet. Sci. Lett., 243, 383-389.

Niu, F. \& Wen, L., 2001. Hemispherical variations in seismic velocity at the top of the Earth's inner core, Nature, 410(6832), 1081-1084.

Ohta, K., Kuwayama, Y., Hirose, K., Shimizu, K. \& Ohishi, Y., 2016. Experimental determination of the electrical resistivity of iron at Earth's core conditions, Nature, 534(7605), 95-98.

O'Rourke, J.G., Korenaga, J. \& Stevenson, D.J., 2017. Thermal evolution of earth with magnesium precipitation in the core, Earth planet. Sci. Lett., 458, 263-272.

O'Rourke, J.G. \& Stevenson, D.J., 2016. Powering earth's dynamo with magnesium precipitation from the core, Nature, 529(7586), 387-389.

Pais, A \& Jault, D., 2008. Quasi-geostrophic flows responsible for the secular variation of the Earth's magnetic field, Geophys. J. Int., 173(2), 421-443.

Poupinet, G., Pillet, R. \& Souriau, A, 1983. Possible heterogeneity of the Earth's core deduced from PKIKP travel times, Nature, 305, 204-206.

Pozzo, M., Davies, C., Gubbins, D. \& Alfè, D., 2012. Thermal and electrical conductivity of iron at Earth's core conditions, Nature, 485, 355-358.

Pozzo, M., Davies, C., Gubbins, D. \& Alfè, D., 2014. Thermal and electrical conductivity of solid iron and iron-silicon mixtures at Earth's core conditions, Earth planet. Sci. Lett., 393, 159-164.

Reaman, D., Colijn, H., Yang, F., Hauser, A \& Panero, W., 2012. Interdiffusion of Earth's core materials to $65 \mathrm{GPa}$ and $2200 \mathrm{~K}$, Earth planet. Sci. Lett., 349, 8-14.

Reaman, D.M., Daehn, G.S. \& Panero, W.R., 2011. Predictive mechanism for anisotropy development in the Earth's inner core, Earth planet. Sci. Lett., 312(3-4), 437-442.

Sha, X. \& Cohen, R., 2011. First-principles studies of electrical resistivity of iron under pressure, J. Phys.: Condens. Matter, 23, 075401, doi:10.1088/0953-8984/23/7/075401.

Sneddon, I., 1960. On some infinite series involving the zeros of Bessel functions of the first kind, in Proceedings of the Glasgow Mathematical Association, Vol. 4, pp. 144-156, Cambridge Univ. Press.

Souriau, A \& Poupinet, G., 1991. The velocity profile at the base of the liquid core from $\mathrm{PKP}(\mathrm{BC}+\mathrm{Cdiff})$ data: an argument in favor of radial inhomogeneity, Geophys. Res. Lett., 18, 2023-2026.

Stacey, F.D. \& Davis, P.M., 2008. Physics of the Earth, Cambridge Univ. Press.
Stern, M.E., 1960. The salt-fountain and thermohaline convection, Tellus, 12(2), 172-175.

Stommel, H., Arons, A.B. \& Blanchard, D., 1956. An oceanographical curiosity: the perpetual salt fountain, Deep Sea Res., 3(2), 152-153.

Sumita, I. \& Olson, P., 1999. A laboratory model for convection in Earth's core driven by a thermally heterogeneous mantle, Science, 286, 15471549.

Tanaka, S., 2012. Depth extent of hemispherical inner core from PKP (DF) and PKP (Cdiff) for equatorial paths, Phys. Earth planet. Inter., 210, $50-62$.

Tanaka, S. \& Hamaguchi, H., 1997. Degree one heterogeneity and hemispherical variation of anisotropy in the inner core from PKP(BC)PKP(DF) times, J. geophys. Res., 102, 2925-2938.

Turner, J.S., 1974. Double-Diffusive Phenomena, Annu. Rev. Fluid Mech., 6, 37-54.

Van Orman, J.A., 2004. On the viscosity and creep mechanism of Earth's inner core, Geophys. Res. Lett., 31, L20606, doi:10.1029/2004GL021209.

Vocadlo, L., 2015. Earths core: iron and iron alloys, in Treatise on Geophysics, Vol. 2, pp. 117-147, ed. ,Schubert, G., Elsevier.

Waszek, L., Irving, J. \& Deuss, A, 2011. Reconciling the hemispherical structure of Earth's inner core with its super-rotation, Nat. Geosci., 4(4), 264-267.

Woodhouse, J.H., Giardini, D. \& Li, X.-D., 1986. Evidence for inner core anisotropy from free oscillations., Geophys. Res. Lett., 13, 1549-1552.

Yoshida, S., Sumita, I. \& Kumazawa, M., 1996. Growth model of the inner core coupled with the outer core dynamics and the resulting elastic anisotropy, J. geophys. Res., 101, 28085-28104.

Zhang, P., Cohen, R. \& Haule, K., 2016. Retraction: effects of electron correlations on transport properties of iron at earth's core conditions, Nature, 536, 112.

\section{APPENDIX: GOVERNING EQUATIONS FOR THE TRANSLATION MODE $(\mathcal{P} \rightarrow 0$ L I M I T)}

\section{A1 Translation rate}

The force balance on the inner core writes

$0=\int_{V}\left(\rho+\rho^{\prime}\right) \mathbf{g} \mathrm{d} V+\int_{S}(\underline{\tau} \cdot \mathbf{n}-P \mathbf{n}) \mathrm{d} S$,

where $\rho$ is the reference inner core density, $\rho^{\prime}$ is density perturbations about $\rho$, and where surface stresses have been decomposed into pressure $P$ and deviatoric stresses $\underline{\tau} \cdot \mathbf{n}$. Neglecting the small radial variations of $\rho$, and writing $\mathbf{g}=-\nabla \Psi$, we can transform the volume integral of $\rho \mathbf{g}$ into a surface integral, which gives

$0=\int_{V} \rho^{\prime} \mathbf{g} \mathrm{d} V-\int_{S}(P+\rho \Psi) \mathrm{d} \mathbf{S}+\int_{S} \underline{\tau} \cdot \mathrm{d} \mathbf{S}$.

Assuming the last integral vanishes (i.e. no net viscous force imposed by the outer core on the inner core and vanishing electromagnetic coupling), the force balance on the inner core comes down to an equilibrium between buoyancy forces associated with density anomalies within the inner core and pressure forces applied by the outer core on the inner core surface. Projecting eq. (A2) on the translation direction, we obtain

$0=\frac{\rho g_{\text {icb }}}{r_{\text {ic }}} \int_{V}\left(\alpha_{T} \Theta+\alpha_{c} \chi\right) r \cos \theta \mathrm{d} V-\int_{S}(P+\rho \Psi) \cos \theta \mathrm{d} S$

with the assumption that the gravity acceleration in the inner core is $\mathbf{g}=-g_{\text {icb }}\left(r / r_{\text {icb }}\right) \mathbf{e}_{r}$. Here $\theta$ is the angle between $\mathbf{r}$ and the translation direction.

Let us denote by $h(\theta)$ the topography of the ICB, defined with respect to a reference isopotential surface that has a mean radius which coincides with the mean inner core radius. In the outer core, 
isopressure surfaces coincide with isopotentials, when averaged on timescales long compared to the timescales of outer core dynamics. The pressure applied by the liquid outer core on the ICB is, therefore, given by $P(h)=P_{0}-\rho_{l} g_{\text {icb }} h$, where $P_{0}$ is the value of the pressure on the reference isopotential. The gravity potential at the ICB is estimated by expanding $\Psi(h, \theta)$ at first order in $h$, which gives $\Psi(h, \theta)=\Psi_{0}+g_{\text {icb }} h+\mathcal{O}\left(h^{2}\right)$, where $\Psi_{0}$ is the value of $\Psi$ on the reference isopotential. Using the above expressions to write $P$ and $\Psi$ at the ICB as functions of $h$ at first order in $h$, eq. (A3) can be written as

$$
\begin{aligned}
0= & \frac{\rho}{r_{\mathrm{ic}}} \int_{V}\left(\alpha_{T} \Theta+\alpha_{c} \chi\right) r \cos \theta \mathrm{d} V-\Delta \rho \int_{S} h \cos \theta \mathrm{d} S, \\
= & 2 \pi \frac{\rho}{r_{\mathrm{ic}}} \int_{0}^{r_{\mathrm{ic}}} \int_{0}^{\pi}\left(\alpha_{T} \Theta+\alpha_{c} \chi\right) r^{3} \cos \theta \sin \theta \mathrm{d} \theta \mathrm{d} r \\
& -2 \pi \Delta \rho r_{\mathrm{ic}}^{2} \int_{0}^{\pi} h \cos \theta \sin \theta \mathrm{d} \theta .
\end{aligned}
$$

We now expand $h, \Theta$ and $\chi$ as series of Legendre polynomials of $\cos \theta$ (which correspond to zonal spherical harmonics):

$h=\sum_{l=0}^{\infty} h_{l} P_{l}(\cos \theta)$

$\Theta=\sum_{l=0}^{\infty} \Theta_{l}(r) P_{l}(\cos \theta)$,

$\chi=\sum_{l=0}^{\infty} \chi_{l}(r) P_{l}(\cos \theta)$

The Legendre polynomials satisfy the orthogonality condition

$$
\int_{0}^{\pi} P_{l}(\cos \theta) P_{m}(\cos \theta) \sin \theta d \theta=\frac{2 \delta_{l m}}{2 l+1}, \quad \forall(l, m) \in \mathbb{N}^{+2},
$$

and since $P_{1}(\cos \theta)=\cos \theta$, the $\theta-$ integrals in eq. (A4) are proportionals to the degree 1 coefficient of their integrand. eq. (A4) reduces to an equation giving the degree 1 component of the topography (which is also equal to the displacement of the inner core along the translation direction) as a functions of integrals of the degree 1 temperature and composition heterogeneities:

$h_{1}=\frac{\rho}{\Delta \rho r_{\mathrm{ic}}^{3}} \int_{0}^{r_{\mathrm{icb}}}\left[\alpha_{T} \Theta_{1}(r)+\alpha_{c} \chi_{1}(r)\right] r^{3} \mathrm{~d} r$.

We can then couple this equation with the phase change law (eq. (10)), giving $V=h_{1} / \tau_{\varphi}$, to obtain an expression for the translation rate as a function of the density heterogeneities within the inner core:

$V=\frac{\rho}{\Delta \rho r_{\mathrm{ic}}^{3} \tau_{\phi}} \int_{0}^{r_{\mathrm{icb}}}\left[\alpha_{T} \Theta_{1}(r)+\alpha_{c} \chi_{1}(r)\right] r^{3} \mathrm{~d} r$.

\section{A2 Legendre polynomials decomposition of the heat and composition conservation equations}

We only allow here for the translation mode, for which the potential temperature and composition fields are symmetric about the translation direction. The equation of transport of heat is then given by eq. (1) in which advective transport is restricted to the translation direction,

$$
\frac{\partial \Theta}{\partial t}+V \mathbf{e}_{x} \cdot \nabla \Theta=\kappa_{T} \nabla^{2} \Theta+S_{T},
$$

which can be recast as

$$
\frac{\partial \Theta}{\partial t}=-V \cos \theta \frac{\partial \Theta}{\partial r}+V \frac{\sin \theta}{r} \frac{\partial \Theta}{\partial \theta}+\kappa_{T} \nabla^{2} \Theta+S_{T}
$$

Defining a new variable $x=\cos \theta$ and substituting in the above equation gives

$$
\frac{\partial \Theta}{\partial t}=-V x \frac{\partial \Theta}{\partial r}+\frac{V}{r}\left(x^{2}-1\right) \frac{\partial \Theta}{\partial x}+\kappa_{T} \nabla^{2} \Theta+S_{T} .
$$

We now decompose $\Theta$ as a sum of Legendre polynomials [eq. (A6)], and take the inner product of eq. (A13) with $P_{l}(x)$, using the orthogonality relation $\int_{-1}^{1} P_{l}^{2}(x) \mathrm{d} x=\frac{2}{2 l+1}$ to obtain:

$$
\begin{aligned}
\frac{2}{2 l+1} \frac{\partial \Theta_{l}}{\partial t}= & -V \int_{-1}^{1} x \frac{\partial \Theta}{\partial r} P_{l}(x) \mathrm{d} x \\
& +\frac{V}{r} \int_{-1}^{1}\left(x^{2}-1\right) \frac{\partial \Theta}{\partial x} P_{l}(x) \mathrm{d} x \\
& +\frac{2}{2 l+1} \kappa_{T} \mathcal{D}_{l} \Theta_{l}+\frac{2}{2 l+1} S_{T} \delta_{0 l},
\end{aligned}
$$

where the operator $\mathcal{D}_{l}$ is defined as

$\mathcal{D}_{l}=\frac{\mathrm{d}^{2}}{\mathrm{~d} r^{2}}+\frac{2}{r} \frac{\mathrm{d}}{\mathrm{d} r}-\frac{l(l+1)}{r^{2}}$

Using the recurrence relation

$x P_{n}=\frac{n+1}{2 n+1} P_{n+1}+\frac{n}{2 n+1} P_{n-1}$

(Abramovich \& Stegun 1965, p. 782), we write the first integral in eq. (A14) as

$$
\begin{aligned}
\int_{-1}^{1} x \frac{\partial \Theta}{\partial r} P_{l}(x) \mathrm{d} x= & \int_{-1}^{1} \frac{\partial \Theta}{\partial r}\left(\frac{l+1}{2 l+1} P_{l+1}+\frac{l}{2 l+1} P_{l-1}\right) \mathrm{d} x \\
= & \frac{l+1}{2 l+1} \frac{2}{2 l+3} \frac{\partial \Theta_{l+1}}{\partial r} \\
& +\frac{l}{2 l+1} \frac{2}{2 l-1} \frac{\partial \Theta_{l-1}}{\partial r}
\end{aligned}
$$

using again the orthogonality relation.

Using the differential relation

$\left(1-x^{2}\right) P_{n}^{\prime}(x)=-n x P_{n}+n P_{n-1}$

$=-\frac{n(n+1)}{2 n+1} P_{n+1}+\frac{n(n+1)}{2 n+1} P_{n-1}$

(Abramovich \& Stegun 1965, p. 783), we write the second integral as

$$
\begin{aligned}
\int_{-1}^{1} & \left(x^{2}-1\right) \frac{\partial \Theta}{\partial x} P_{l}(x) \mathrm{d} x \\
& =\sum_{n=0}^{\infty} \Theta_{n} \int_{-1}^{1}\left(x^{2}-1\right) \frac{\partial P_{n}}{\partial x} P_{l}(x) \mathrm{d} x \\
& =\sum_{n=0}^{\infty} \Theta_{n} \int_{-1}^{1}\left(\frac{n(n+1)}{2 n+1} P_{n+1}-\frac{n(n+1)}{2 n+1} P_{n-1}\right) P_{l}(x) \mathrm{d} x \\
& =\frac{2 l(l-1)}{(2 l-1)(2 l+1)} \Theta_{l-1}-\frac{2(l+2)(l+1)}{(2 l+1)(2 l+3)} \Theta_{l+1} .
\end{aligned}
$$


Combining eqs (A17) and (A20), we finally obtain the following expression for the potential temperature transport equation:

$$
\begin{aligned}
\frac{\partial \Theta_{l}}{\partial t}= & -V \frac{l+1}{2 l+3}\left(\frac{l+2}{r}+\frac{\partial}{\partial r}\right) \Theta_{l+1} \\
& +V \frac{l}{2 l-1}\left(\frac{l-1}{r}-\frac{\partial}{\partial r}\right) \Theta_{l-1} \\
& +\kappa_{T} \mathcal{D}_{l} \Theta_{l}+S_{T} \delta_{0 l},
\end{aligned}
$$

A similar equation holds for the evolution of composition.

\section{A3 Growth rate of the translation instability}

\section{A3.1 Dispersion equation}

We now estimate the growth rate of the instability, by linearizing the heat and composition transport equations, which, similarly as done in Section 4.2.1, we write as

$\frac{\partial \Theta_{1}}{\partial t}=-V \frac{\partial \Theta_{0}}{\partial r}+\mathcal{D}_{1} \Theta_{1}$,

$\frac{\partial \chi_{1}}{\partial t}=-V \frac{\partial \chi_{0}}{\partial r}+\frac{1}{L e} \mathcal{D}_{1} \chi_{1}$

The translation rate is given by

$V=\int_{0}^{1}\left(\mathcal{R}_{\Theta} \Theta_{1}+\frac{\mathcal{R}_{\chi}}{L e} \chi_{1}\right) r^{3} \mathrm{~d} r$

The degree 1 components $\Theta_{1}$ and $\chi_{1}$ of the potential temperature and composition fields are expanded as series of spherical Bessel functions of the first kind and order $1, j_{1}$,

$\Theta_{1}=\sum_{i=1}^{\infty} A_{i}^{\Theta} j_{1}\left(\alpha_{1 i} r\right) \mathrm{e}^{\sigma t}$

$\chi_{1}=\sum_{i=1}^{\infty} A_{i}^{\chi} j_{1}\left(\alpha_{1 i} r\right) \mathrm{e}^{\sigma t}$,

where $\sigma$ is the growth rate and $\alpha_{1 i}$ is the $i$ th zero of $j_{1}$. The functions $r \rightarrow j_{1}\left(\alpha_{1 i} r\right)$ for $i=1,2, \ldots, \infty$ form a complete set of orthogonal functions on $[0,1]$, and satisfy the orthogonality relation

$$
\int_{0}^{1} r^{2} j_{1}\left(\alpha_{1 i} r\right) j_{1}\left(\alpha_{1 j} r\right) \mathrm{d} r=\delta_{i, j} \frac{\sin ^{2} \alpha_{1 j}}{2 \alpha_{1 j}^{2}}
$$

(Chandrasekhar 1961, Chapter 6, section 59). The functions $r \rightarrow$ $j_{1}\left(\alpha_{1 i} r\right)$ are eigenfunctions of the operator $\mathcal{D}_{1}$ [eq. (21)], such that

$\mathcal{D}_{1} j_{1}\left(\alpha_{1 i} r\right)=-\alpha_{1 i}^{2} j_{1}\left(\alpha_{1 i} r\right)$.

Inserting eqs (A25) and (A26) into eq. (A24) gives

$V=\sum_{i=1}^{\infty}\left(\mathcal{R}_{\Theta} A_{i}^{\Theta}+\frac{\mathcal{R}_{\chi}}{L e} A_{i}^{\chi}\right) \mathrm{e}^{\sigma t} \int_{0}^{1} j_{1}\left(\alpha_{1 i} r\right) r^{3} \mathrm{~d} r$,

$=-\sum_{i=1}^{\infty} \frac{\sin \alpha_{1 i}}{\alpha_{1 i}^{2}}\left(\mathcal{R}_{\Theta} A_{i}^{\Theta}+\frac{\mathcal{R}_{\chi}}{L e} A_{i}^{\chi}\right) \mathrm{e}^{\sigma t}$,

where eq. (A33) of Deguen et al. (2013) has been used to calculate the integral on RHS of eq. (A29).

The coefficients $A_{i}^{\Theta}$ and $A_{i}^{\chi}$ are obtained by inserting eqs (A25) and (A26) into eqs (A22) and (A23)

$\sum_{i} A_{i}^{\Theta}\left(\sigma+\alpha_{1 i}^{2}\right) j_{1}\left(\alpha_{1 i} r\right)=-V \frac{\partial \Theta_{0}}{\partial r}$, $\sum_{i} A_{i}^{\chi}\left(\sigma+\frac{\alpha_{1 i}^{2}}{L e}\right) j_{1}\left(\alpha_{1 i} r\right)=-V \frac{\partial \chi_{0}}{\partial r}$.

before multiplying with $j_{1}\left(\alpha_{1 i} r\right)$ and applying the orthogonality relation eq. (A27) to obtain

$A_{i}^{\Theta}=-\frac{2 \alpha_{1 i}^{2} V}{\left(\sigma+\alpha_{1 i}^{2}\right) \sin ^{2} \alpha_{1 i}} \int_{0}^{1} \frac{\partial \Theta_{0}}{\partial r} j_{1}\left(\alpha_{1 i} r\right) r^{2} \mathrm{~d} r$,

$A_{i}^{\chi}=-\frac{2 \alpha_{1 i}^{2} V}{\left(\sigma+\alpha_{1 i}^{2} / L e\right) \sin ^{2} \alpha_{1 i}} \int_{0}^{1} \frac{\partial \chi_{0}}{\partial r} j_{1}\left(\alpha_{1 i} r\right) r^{2} \mathrm{~d} r$

Inserting the above expressions for $A_{i}^{\Theta}$ and $A_{i}^{\chi}$ into eq. (A30) gives the following equation for $\sigma$ :

$$
\begin{aligned}
& \mathcal{R}_{\Theta} \sum_{i=1}^{\infty} \frac{2}{\left(\sigma+\alpha_{1 i}^{2}\right) \sin \alpha_{1 i}} \int_{0}^{1} \frac{\partial \Theta_{0}}{\partial r} j_{1}\left(\alpha_{1 i} r\right) r^{2} \mathrm{~d} r \\
& \quad+\mathcal{R}_{\chi} \sum_{i=1}^{\infty} \frac{2}{\left(L e \sigma+\alpha_{1 i}^{2}\right) \sin \alpha_{1 i}} \int_{0}^{1} \frac{\partial \chi_{0}}{\partial r} j_{1}\left(\alpha_{1 i} r\right) r^{2} \mathrm{~d} r=1 .
\end{aligned}
$$

With the base state profiles

$\Theta_{0}=1-r^{2}$,

$\chi_{0}=\frac{\frac{3}{2} r_{\mathrm{ic}}^{*}\left(1-r^{2}\right)-\left(1-r^{3}\right)}{\frac{3}{2} r_{\mathrm{ic}}^{*}-1}$,

we obtain

$\int_{0}^{1} \frac{\partial \Theta_{0}}{\partial r} j_{1}\left(\alpha_{1 i} r\right) r^{2} \mathrm{~d} r=2 \frac{\sin \alpha_{1 i}}{\alpha_{1 i}^{2}}$

$\int_{0}^{1} \frac{\partial \chi_{0}}{\partial r} j_{1}\left(\alpha_{1 i} r\right) r^{2} \mathrm{~d} r=2 \frac{\sin \alpha_{1 i}}{\alpha_{1 i}^{2}} \mathcal{F}\left(r_{\mathrm{ic}}^{*}, \alpha_{1 i}\right)$

where

$\mathcal{F}\left(r_{\mathrm{ic}}^{*}, \alpha_{1 i}\right)=\frac{1-\frac{4}{\alpha_{1 i}^{2}}\left(1+\frac{2}{\alpha_{1 i}^{2}}-\frac{2}{\alpha_{1 i} \sin \alpha_{1 i}}\right)-r_{\mathrm{ic}}^{*}}{2 / 3-r_{\mathrm{ic}}^{*}}$.

When deriving eqs (A38) and (A39), we have made use of the relations

$\int_{0}^{1} j_{1}\left(\alpha_{1 i} r\right) r^{3} \mathrm{~d} r=-\frac{\sin \alpha_{1 i}}{\alpha_{1 i}^{2}}$

$\int_{0}^{1} j_{1}\left(\alpha_{1 i} r\right) r^{4} \mathrm{~d} r=-\frac{\left(\alpha_{1 i}^{4}-4 \alpha_{1 i}^{2}-8\right) \sin \alpha_{1 i}+8 \alpha_{1 i}}{\alpha_{1 i}^{6}}$,

[eq. (A41) is demonstrated in Deguen et al. (2013); eq. (A42) can be verified by direct integration].

Finally, inserting eqs (A38) and (A39) into eq. (A35) gives the following dispersion equation

$\mathcal{R}_{\Theta} \sum_{i=1}^{\infty} \frac{4}{\alpha_{1 i}^{2}\left(\sigma+\alpha_{1 i}^{2}\right)}+\mathcal{R}_{\chi} \sum_{i=1}^{\infty} \frac{4 \mathcal{F}\left(r_{\mathrm{ic}}^{*}, \alpha_{1 i}\right)}{\alpha_{1 i}^{2}\left(\operatorname{Le} \sigma+\alpha_{1 i}^{2}\right)}=1$. 


\section{A3.2 Marginal stability}

The critical values of $\mathcal{R}_{\Theta}$ and $\mathcal{R}_{\chi}$ can be obtained by setting $\sigma=0$ into eq. (A43), which then writes

$\frac{\mathcal{R}_{\Theta}}{\mathcal{R}_{\Theta, \mathrm{cr}}}+\frac{\mathcal{R}_{\chi}}{\mathcal{R}_{\chi, \mathrm{cr}}}=1$

where

$\mathcal{R}_{\Theta, \mathrm{cr}}=\frac{1}{4}\left[\sum_{i=1}^{\infty} \frac{1}{\alpha_{1 i}^{4}}\right]^{-1}$,

$\mathcal{R}_{\chi, \mathrm{cr}}=\frac{1}{4}\left[\sum_{i=1}^{\infty} \frac{\mathcal{F}\left(r_{\mathrm{ic}}^{*}, \alpha_{1 i}\right)}{\alpha_{1 i}^{4}}\right]^{-1}$,

which can be used to verify the consistency of the dispersion eq. (A43) with the results obtained in Section 4.2.1 for the marginal state. Using the results of Sneddon (1960) that $\sum_{i=1}^{\infty} \alpha_{1 i}^{-4}=1 / 350$, we obtain

$\mathcal{R}_{\Theta, \text { cr }}=\frac{1}{4}\left(\sum_{i=1}^{\infty} \frac{1}{\alpha_{1 i}^{4}}\right)^{-1}=\frac{175}{2}$

as was found in Section 4.2.1. It can be verified by computing the sum in eq. (A46) that is consistent with the expression

$\mathcal{R}_{\chi, c r}=80 \frac{\frac{3}{2} r_{\mathrm{ic}}^{*}-1}{\frac{48}{35} r_{\mathrm{ic}}^{*}-1}$

found in Section 4.2.1.

\section{A3.3 Growth rate}

To estimate the growth rate of the translation instability, the dispersion eq. (A43) can be solved numerically (with truncated sums) for given values of $\mathcal{R}_{\Theta}$ and $\mathcal{R}_{\chi}$. The results are shown as thick continuous lines of Fig. 1. An analytical approximation of the growth rate can be obtained as follows.
In the limit of $L e \gg 1$, diffusion of the compositional field can be neglected. In this limit the composition equation (A23) then reduces to

$\frac{\partial \chi_{1}}{\partial t}=-V \frac{\partial \chi_{0}}{\partial r}$,

from which we obtain

$\chi_{1}=-\frac{V}{\sigma} \frac{\partial \chi_{0}}{\partial r}$

by noting that by construction $V \propto \mathrm{e}^{\sigma t}$.

We further assume that the temperature field is in a quasi-steady state in which the production of degree 1 through translation of the degree 0 is almost exactly balanced by diffusion of the degree 1 . This amounts to assumption that the timescale of instability growth $1 / \sigma$ is large compared to the timescale of diffusion of a degree one heterogeneity. Under this assumption, eq. (A23) reduces to

$0=-V \frac{\partial \Theta_{0}}{\partial r}+\mathcal{D}_{1} \Theta_{1}$

With $\Theta_{0}=1-r^{2}$, the solution is

$\Theta_{1}=\frac{V}{5}\left(r-r^{3}\right)$.

Inserting eqs (A50) and (A52) into eq. (A24) and evaluating the integrals gives

$V=\frac{\mathcal{R}_{\Theta}}{175 / 2} V+\frac{1 / 3-2 / 5 r_{\mathrm{ic}}^{*}}{2 / 3-r_{\mathrm{ic}}^{*}} \frac{\mathcal{R}_{\chi}}{L e} \frac{V}{\sigma}$,

from which we obtain

$\sigma=\frac{1 / 3-2 / 5 r_{\mathrm{ic}}^{*}}{2 / 3-r_{\mathrm{ic}}^{*}} \frac{\mathcal{R}_{\chi} / L e}{1-\frac{\mathcal{R}_{\Theta}}{175 / 2}}$.

Fig. 1 shows both the numerical solution of (A43) and the approximate solution given by eq. (A54). The agreement is excellent at small values of $\sigma$, and fair though not perfect at large values of $\sigma$, a consequence of the thermal quasi-steady state assumption becoming less justified. 